Mon. Not. R. Astron. Soc. 000,1-?? (2016) Printed 23 May $2022 \quad$ (MN LATEX style file v2.2)

\title{
Investigation of open clusters based on IPHAS and APASS survey data
}

\author{
A. K. Dambis ${ }^{1 \star}$, E. V. Glushkova ${ }^{1,2}$, L. N. Berdnikov ${ }^{1,3}$, Y. C. Joshi ${ }^{4}$ \\ A. K. Pandey ${ }^{4}$ \\ ${ }^{1}$ Sternberg Astronomical Institute, Lomonosov Moscow State University, 13, Universitetskii prospect, Moscow, 119992, Russia \\ ${ }^{2}$ Physics Faculty, Lomonosov Moscow State University, 1, bld.2, Leninskie Gory, Moscow, 119991, Russia \\ ${ }^{3}$ Astronomy and Astrophysics Research division, Entoto Observatory and Research Center, P.O.Box 8412, Addis Ababa, Ethiopia \\ ${ }^{4}$ Aryabhatta Research Institute of Observational Sciences, Nainital 263002, India
}

Accepted 2016 MONTH XX.

\begin{abstract}
We adapt the classical $Q$-method based on a reddening-free parameter constructed from three passband magnitudes to the filter set of IPHAS survey and combine it with the maximum-likelihood-based cluster parameter estimator by Navlor \& Jeffries (2006) to determine the extinction, heliocentric distances, and ages of young open clusters using $\mathrm{H}_{\alpha}$ ri data. The method is also adapted for the case of significant variations of extinction across the cluster field. Our technique is validated by comparing the colour excesses, distances, and ages determined in this study with the most bona fide values reported for the 18 well-studied young open clusters in the past and a fairly good agreement is found between our extinction and distance estimates and earlier published results, although our age estimates are not very consistent with those published by other authors. We also show that individual extinction values can be determined rather accurately for stars with $(r-i)>0.1$. Our results open up a prospect for determining a uniform set of parameters for northern clusters based on homogeneous photometric data, and for searching for new, hitherto undiscovered open clusters.
\end{abstract}

Key words: methods: observational - techniques: photometric - general:open clusters and associations : fundamental parameters

\section{INTRODUCTION}

Galactic clusters have been popular among the researchers for several centuries, particularly open clusters (OC), because they are the "building blocks" that make up our Milky Way Galaxy. Large-scale sky surveys have brought about the discovery of many hitherto unknown open clusters, which soon outnumbered those already known over the past 150 years. Currently, about 4000 open clusters are known and the cluster sample within about $1.7-1.8 \mathrm{kpc}$ from the Sun can be considered to be almost complete (Joshi 2007; Joshi et al. 2016). However, a homogeneous catalogue of fundamental open-cluster parameters, primarily colour excesses, ages, and heliocentric distances, is needed for such a rich subsystem to be used for the study of the structure, kinematics, and evolution of the Galactic disk or for solving the problems of star formation. There are ongoing attempts to create such a catalogue. Some authors try to produce the most extensive possible catalogue of cluster pa-

* E-mail: dambis@yandex.ru rameters by compiling published data (Dias et al. 2002) or by determining the cluster parameters based on published observational data (Kharchenko et al. 2013), however, the reported parameters in these compilations seem to be insufficiently accurate for the study of the properties of the Galactic disk (Netopil et al. 2015). Other researchers determined the cluster parameters from their own observational data, however, such catalogues are rather small and may take several decades to complete (Bragaglia and Tosi 2006; Sung et al. 2013; Caetano et al. 2015). The main problem with the determination of fundamental cluster parameters like age, distance, and colour excess is that an empirical ZAMS or isochrone cannot always be unambiguously fitted to the cluster main sequence on colour-magnitude diagrams. Despite the development of new techniques and algorithms for the determination of the basic parameters of open clusters (Navlor 2009; Dias et al. 2012; Popescu et al. 2014; Perren et al. 2015), no breakthrough has been achieved so far to prepare a uniform and accurate catalogue of cluster parameters. The most bona fide estimates of colour excess $E_{B-V}$ can be obtained by fitting ZAMS to the dwarf se- 
quence on the (U-B, B-V) colour-colour diagram. However, the intrinsic colour line for late-type stars in colour-colour diagrams runs almost parallel to the reddening line, therefore this method is applicable only to relatively young clusters. Since most of the large surveys lack U-band photometry, primarily because it requires long signal integration times, hence reddening cannot be determined. However, we show below that there is another kind of colour-colour diagrams based on already available observational data that can be used to unambiguously and rather accurately determine the colour excesses of young clusters. In the present study we use $r, i$, and $\mathrm{H}_{\alpha}$-band magnitudes from IPHAS DR2 survey (Barentsen et al. 2014; Drew et al. 2005) to determine colour excesses of some poorly investigated clusters.

\section{DATA}

As we already pointed out above, our source of photometry is the INT Photometric $\mathrm{H}_{\alpha}$ Survey of the Northern Galactic Plane (IPHAS), which is based on observations made using wide field camera (WFC) on the $2.5 \mathrm{~m}$ Isaac Newton Telescope (INT) with Sloan $r$ - and $i$-band filters and $\mathrm{H}_{\alpha}$ narrowband filter (Drew et al. 2005; Gonzales-Solares et al. 2008). The photometric data for cluster stars used in this study were adopted from the latest Data release 2 of the survey catalogue, which covers the entire northern Galactic plane and gives $r, i$, and $\mathrm{H}_{\alpha}$ band magnitudes for about 219 million sources covering Galactic longitudes $1=30^{\circ}-215^{\circ}$ and latitudes $|b|<5^{\circ}$ down to a limiting magnitude of $21.2 \mathrm{mag}$, $20.0 \mathrm{mag}$, and $20.3 \mathrm{mag}$ in the $\mathrm{r}, \mathrm{i}$, and $\mathrm{H}_{\alpha}$ filters, respectively. (Barentsen et al. 2014).

\section{METHOD}

\subsection{Basic ideas}

Sale et al. (2014) used IPHAS photometry to construct a 3D extinction map in the northern part of the Galactic plane. Their procedure was based on the fact that $\left(\mathrm{r}-\mathrm{H}_{\alpha}\right)$ colour index depends primarily on the equivalent width of the $\mathrm{H}_{\alpha}$ line hence on the effective temperature of the source. Their extinction map has an angular resolution and distance sampling of 10 arcmin and $100 \mathrm{pc}$, respectively, and gives the distribution of monochromatic extinction A0 at $5495 \AA$. Sale et al. (2014) constructed their map using the data for 38 million stars for each of which they determined the distance, reddening, mass, surface gravity, and effective temperature. However, according to the above authors, the calculation of all these parameters are subjected to a set of priors as well as on a survey selection function and should be used with much caution and only as approximate estimates of the real parameters. It is also important that Sale et al. (2014) actually treat the overall extinction distribution (and hence the extinction distribution in open clusters) as somewhat smoothed, albeit hierarchical, with a limited sampling mentioned above (10 arcmin and $100 \mathrm{pc}$, respectively) and ignore the existence of compact groups of stars with small spatial and age dispersion. The technique proposed in this paper, on the contrary, is designed especially for open clusters and uses their compact size in the physical space and in the space of ages and interstellar-extinction (we first assume it to be sufficiently uniform for all cluster members and defer the analysis of the variable-extinction case to Section 3.2).

We use somewhat modified colour-colour diagrams based on the IPHAS data. Our idea is to adapt the classical Q-method (Johnson \& Morgan 1953) and construct a reddening-free photometric index based on the $r, i$, and $\mathrm{H}_{\alpha}$ magnitudes provided by the survey. Given three different passbands we have two independent colour indices (e.g., $r-\mathrm{H}_{\alpha}$ and $r-i$ ) and only one possible reddeningfree index (up to an arbitrary linear transformation) linearly depending on the survey magnitudes: $\mathrm{H}_{\alpha}$ index = $\left(r-H_{\alpha}\right)-\left(E_{r-H \alpha} / E_{r-i}\right)(r-i)=\left(r-H_{\alpha}\right)-0.245(r-i)=$ $0.755 r+0.245 i-H_{\alpha}$, where we adopt $\left(E_{r-H \alpha} / E_{r-i}\right)=$ 0.245 in accordance with the Cardelli et al. (1989) reddening law. The resulting index has simple physical meaning: linear combination $0.755 r+0.245 i$ interpolates between $r$ - and $i$ band magnitudes and sort of simulates a very broad band $H_{\alpha}$ magnitude, and hence $0.755 r+0.245 i-H_{\alpha}$ serves as an extinction-independent measure of the $H_{\alpha}$ line strength (with the reverse sign) similar to the $\beta$ index of Stroemgren system, which characterises the strength of the $\mathrm{H}_{\beta}$ absorption line. Hydrogen line strengths are known to be the highest in stars of spectral type A2 (McBride et al. 2008) and hence $\mathrm{H}_{\alpha}$ index has a minimum at the corresponding color index. We therefore use the $\left(0.755 \mathrm{r}+0.245 \mathrm{i}-\mathrm{H}_{\alpha}, r-i\right)$ diagram as our chief extinction-measuring tool in this study. By construction, the reddening lines in the resulting diagrams are parallel to the horizontal axis.

As expected, the corresponding most recent $\left(r-i, \mathrm{H}_{\alpha}\right.$ index) theoretical isochrones constructed in the Padova system (Chen et al. 2014, 2015; Tang et al. 2014) all have a minimum at the same colour excess $(r-i)$ for all ages spanning from $\log (t)=6.0$ to $\log (t)=8.5$. Fig. 1 shows $(r-$ $i, \mathrm{H}_{\alpha}$ index) colour-colour diagram for the solar-metallicity isochrones with ages in the above interval. A reddening line is shown in the top left corner. Note that the minima of all isochrones are located at the same horizontal coordinate, but are somewhat shifted in the vertical direction. Table 1 lists the $(r-i)_{\min }$ values for solar-metallicity models as well as for models with $[\mathrm{Fe} / \mathrm{H}]=-0.5$ and $[\mathrm{Fe} / \mathrm{H}]=+0.5$ (note that in the set of recent Padova stellar evolution models used here the solar distribution of heavy elements is adopted from the by Caffau et al. (2011), corresponding to a Suns metallicity $Z=0.0152)$. As is evident from the table, the abscissa of the minimum appears to be highly invariant not only over a broad age interval, but also highly stable against metallicity variations: the mean $(r-i)_{\min }$ value is equal to +0.026 mag with a standard deviation of 0.004 (per isochrone) and the maximum deviation does not exceed 0.006. The fact that for $(r-i)$ lower than 0.5 metallicity and age have practically no effect on the observed colors is well known, however, we would nevertheless like to emphasize this point quantitatively to show the robustness of our extinction estimates and demonstrate that the position of the minimum of the $\mathrm{H}_{\alpha}$ index vs $(r-i)$ curve is an excellent reddening indicator for clusters that is practically insensitive to cluster age and metallicity. Older isochrones have no left wing (the part of the curve with intrinsic colour index $\left.(r-i)_{0}<0.0\right)$ and that is why we do not show them here. This is because, as we already pointed out above, $\mathrm{H}_{\alpha}$ absorption line reaches maximum strength in the spectra of A2-type stars, 


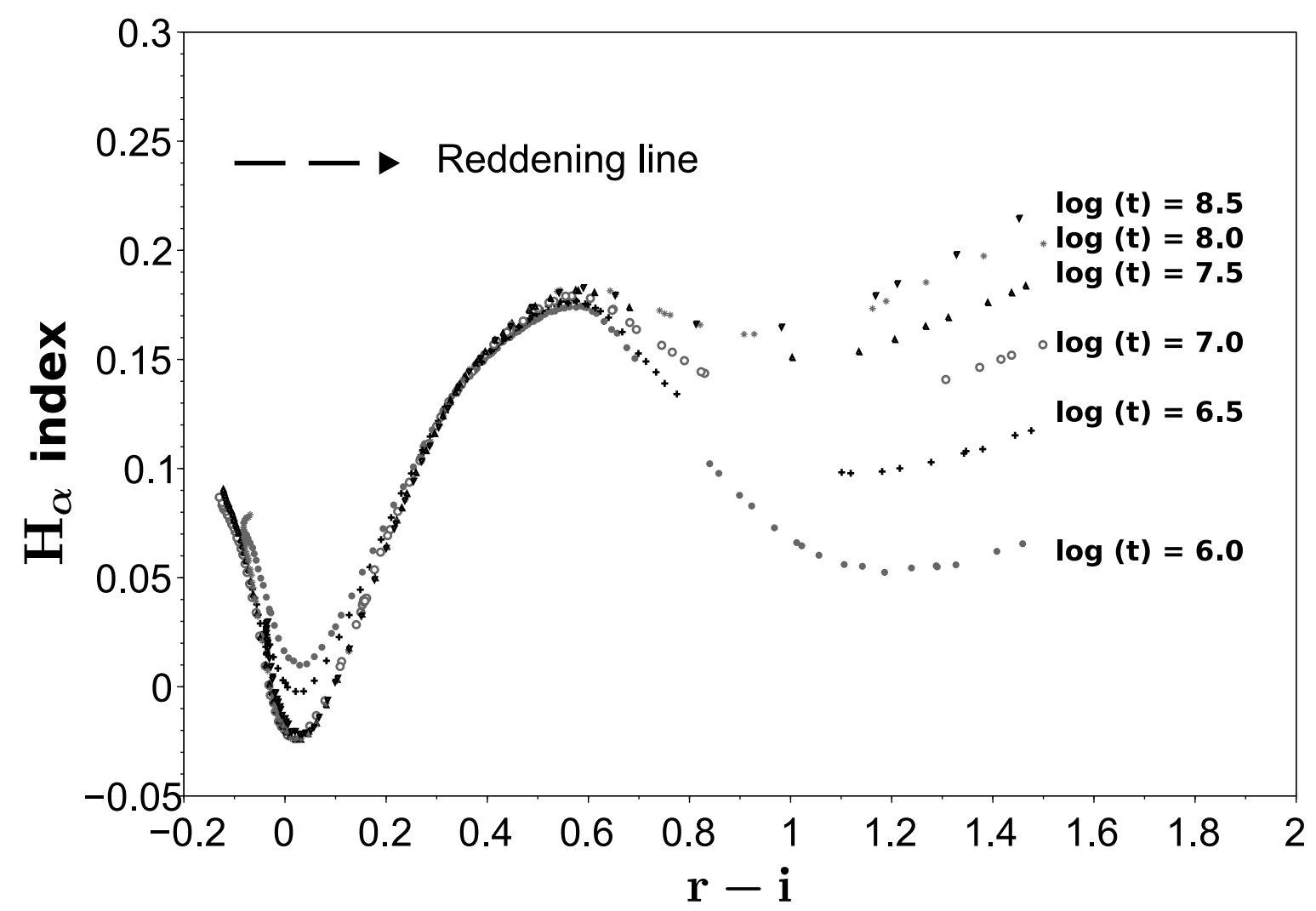

Figure 1. Padova isochrones on the $\left(r-i, \mathrm{H}_{\alpha}\right.$ index $)$ colour-colour diagram.

which are absent in the main sequences of clusters older than $\log (t)=8.5$.

Hence if a cluster contains enough early A-type mainsequence stars and extinction variations across it are not significant enough (the case of highly variable reddening is addressed in Section 3.2), its average $E_{r-i}$ colour excess can theoretically be easily determined by fitting the cluster MS with an isochrone of any age in the $\log (t)=6.0-8.5$ interval simply by shifting the latter along the horizontal axis. We illustrate this in Fig. 2 where we show the $\left(r-i, \mathrm{H}_{\alpha}\right.$ index $)$ diagram for the cluster NGC 7790 with the superimposed $\log (t)=7.2$ isochrone shifted horizontally by $0.38 \mathrm{mag}$. Note, however, that in practice a vertical offset (in the $\mathrm{H}_{\alpha}$ index) has also to be applied because, although the shape of the observed diagram matches fairly well the theoretical curve, the observed $\mathrm{H}_{\alpha}$ index values are located above the theoretical line, which runs about 0.049 below the lower envelope of the observed points. As we show below, this is a common behaviour and the vertical offsets of the lower envelope are more or less the same for all clusters. This offset is most likely due to some systematic zero point mismatches between the color indices as represented by the actual IPHAS catalogue and the corresponding theoretically computed colors.

Now, having determined the colour excess $E_{r-i}$ of a cluster, we can proceed with estimating such fundamental parameters as the distance and age. To this end, we use the $(r-i)-r$ diagram and fit an appropriate theoretical isochrone by shifting it horizontally by the already known $E_{r-i}$ and vertically by the unknown apparent $r$-band distance modulus, $D M_{r}=r-M_{r}$. The true distance modulus is then determined as $D M_{0}=D M_{r}-3.98 E_{r-i}$ and the distance in $\mathrm{kpc}$ as $d=10^{0.2\left(D M_{0}-10\right)}$. Note, however, that whereas the cluster distance can be inferred rather accurately, IPHAS data does not allow age to be determined reliably enough for most of the clusters because stars brighter than $r=13 \mathrm{mag}, i=12 \mathrm{mag}$ are saturated in the IPHAS survey and their magnitudes are subject to systematic errors (Barentsen et al. 2014). As a result, the MS turnoff, which is the region where age differences are most conspicuous, becomes unavailable. To partially mitigate this effect, we use $r i$ photometry of the APASS survey Henden \& Munari 2014; Henden et al. 2016) for such stars, which we transform to the IPHAS photometric systems via the following equations (Barentsen et al. 2014):

$$
\begin{aligned}
& r_{\text {IPHAS }}=r_{\text {APASS }}-0.121+0.032(r-i)_{\text {APASS }} \\
& i_{\text {IPHAS }}=i_{\text {APASS }}-0.364+0.006(r-i)_{\text {APASS }}
\end{aligned}
$$

In this way we push the saturation limit brightwards by $\sim 3$ mag. We illustrate this in Fig. 3 where we show the $(r-i)-r$ diagram for the cluster NGC 7790 with the superimposed $\log (t)=7.81$ isochrone shifted horizontally by $E_{r-i}=0.38 \mathrm{mag}$ and vertically by $D M_{r}=13.90$. The resulting true distance modulus estimate of the cluster is 


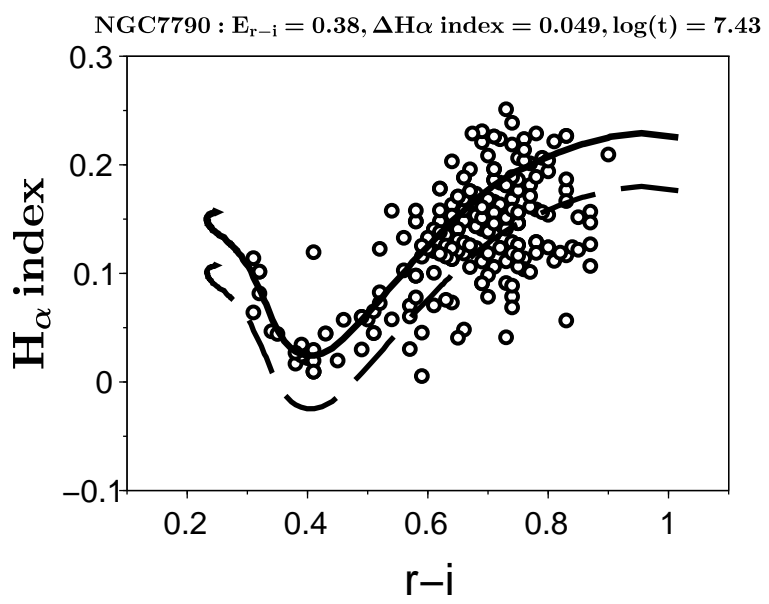

Figure 2. Colour-colour diagram for stars within 3 arcmin of the NGC 7790 cluster centre and the superimposed log $(t)=7.2$ isochrone shifted by $0.38 \mathrm{mag}$ along the horizontal axis (the thin dashed curve) and the same isochrone shifted by $0.38 \mathrm{mag}$ along the horizontal axis and by $\Delta \mathrm{H}_{\alpha}$ index $=+0.049$ along the vertical axis (the thick solid curve).

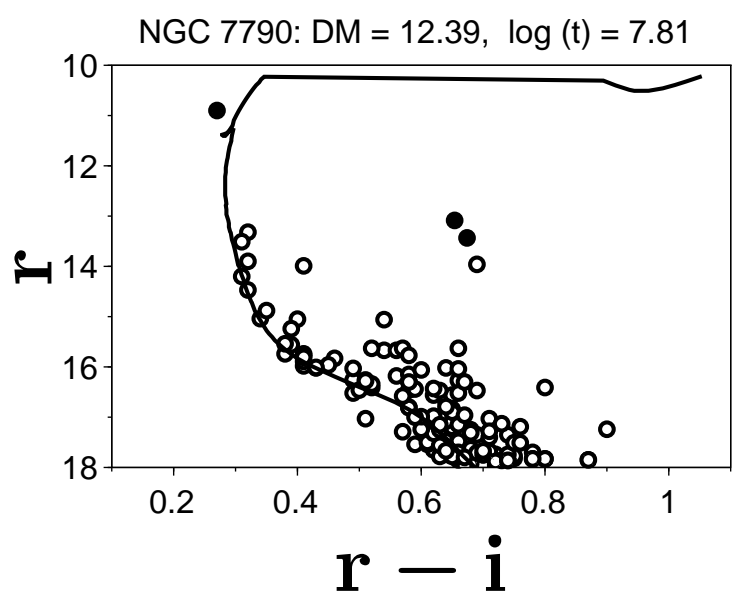

Figure 3. The $(r-i, r)$ colour-magnitude diagram for stars within 3 arcmin from the centre of the NGC 7790 cluster with the superimposed $\log (t)=7.81$ isochrone shifted by 0.38 along the horizontal axis and by $13.90 \mathrm{mag}$ along the vertical axis (the solid curve). The open circles represent unsaturated stars with IPHAS data and the filled circles, stars with APASS ri photometry converted to the IPHAS system in accordance with Eqs. 1] and 2 Given the above colour excess estimate $E_{r-i}=0.38 \mathrm{mag}$ the true distance modulus is $(m-M)_{0}=12.39$, which corresponds to the distance of $d=3006 \mathrm{pc}$.

$D M_{0}=D M_{r}-3.98 E_{r-i}=11.800$, which corresponds to the distance of $3006 \mathrm{pc}$.

The $\left(\mathrm{H}_{\alpha}\right.$ index, $\left.M_{r}\right)$ isochrones with ages no greater than $\log (t)=8.5$ also have an interesting feature: a characteristic leftward tip. Fig. 4 shows the theoretical isochrones $\log (t)=6.0,6.5,7.0,7.5,8.0$, and 8.5 in the $M_{r}$ vs $\mathrm{H}_{\alpha}$ index plane. The tip locations of all isochrones with ages $\log (t)=7.0$ or older have practically the same horizontal and, what is important, vertical coordinate. The apparent (a)

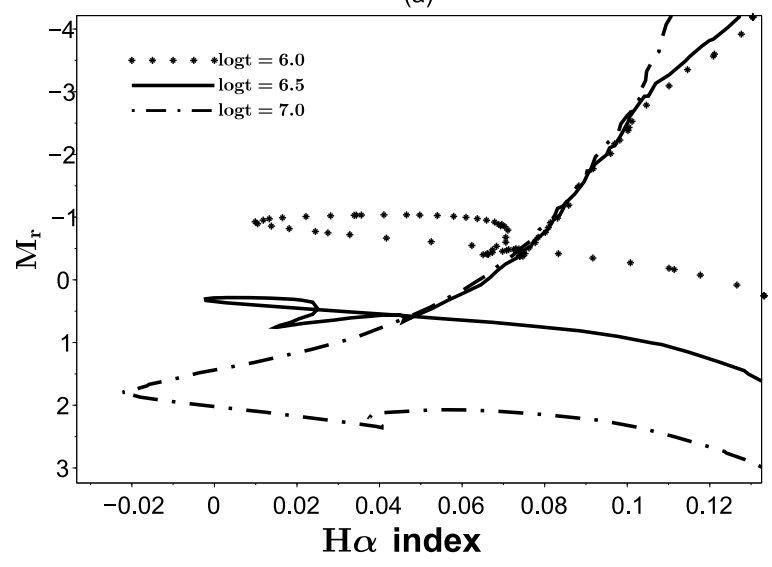

(b)

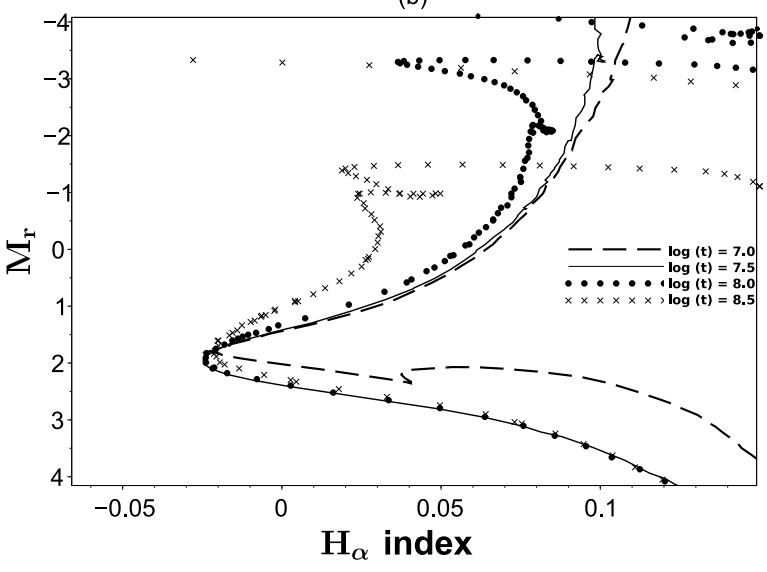

Figure 4. The $\left(\mathrm{H}_{\alpha}\right.$ index, $\left.M_{r}\right)$ diagram for isochrones: (a) $\log (t)=6.0,6.5,7.0$ and $(\mathrm{b}) \log (t)=7.0,7.5,8.0$, and 8.5.

distance modulus $(m-M)_{r}$ of a comparatively young cluster can be determined by shifting the cluster main sequence on the $\left(\mathrm{H}_{\alpha}\right.$ index, $\left.M_{r}\right)$ diagram to fit the isochrone (note that it should be accompanied by a shift in the $\mathrm{H}_{\alpha}$ index to correct for a small inconsistency between the theoretical and observed colors). The distance to the cluster can then be determined from this distance modulus and the colour excess earlier determined from the $\left(r-i, \mathrm{H}_{\alpha}\right.$ index $)$ diagram. Fig. 5 shows the $\left(\mathrm{H}_{\alpha}\right.$ index, $\left.r\right)$ "colour-magnitude" diagram for the cluster NGC 7790 superimposed with the $\log (t)=7.81$ isochrone shifted by $13.74 \mathrm{mag}$ along the vertical axis and by $\Delta \mathrm{H}_{\alpha}$ index $=+0.043$ along the horizontal axis. Thus the distance to the cluster is equal to $2793 \mathrm{pc}$, which more or less agrees with the $2944 \mathrm{pc}$ estimate listed in the catalogue by (Dias et al. 2002) although is appreciably shorter than the 3548 pc estimate by Phelps and Janes (1994).

\subsection{The case of non-uniform extinction}

So far, we assumed that reddening is more or less uniform across the cluster and used the $\left(r-i, \mathrm{H}_{\alpha}\right.$ index $)$ diagram to determine it. This, however, is by no means always the case, and we therefore have to adapt our method to make it applicable to clusters with variable extinction. Our solution is to 


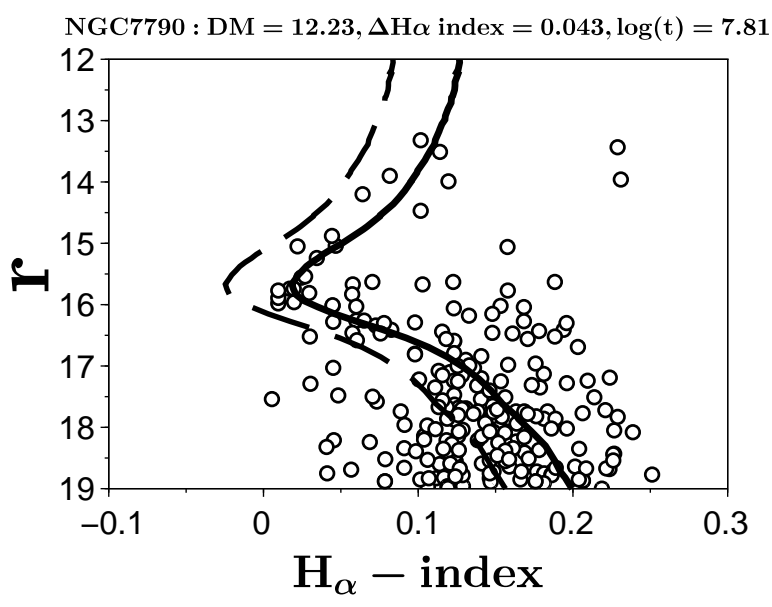

Figure 5. The $\left(\mathrm{H}_{\alpha}\right.$ index, $\left.r\right)$ "colour-magnitude" diagram for stars within 3 arcmin from the centre of the NGC 7790 cluster with the superimposed $\log (t)=7.81$ isochrone shifted by 13.74 mag along the vertical axis (the dashed curve) and the same isochrone shifted by $13.74 \mathrm{mag}$ along the vertical axis and by $\Delta \mathrm{H}_{\alpha}$ index $=+0.043$ along the horizontal axis (the solid curve). Given the above colour excess estimate $E_{r-i}=0.38 \mathrm{mag}$ the true distance modulus is $(m-M)_{0}=12.23$, which corresponds to the distance of $d=2793 \mathrm{pc}$.

operate with extinction-independent quantities exclusively, and our tool of choice in this case is the $\left(\mathrm{H}_{\alpha}\right.$ index, $\left.W_{r i}\right)$ diagram, where $W_{r i}=r-3.98(r-i)$ is extinction-independent Wesenheit index. Fig. 6] shows the theoretical isochrones for $\log (t)=6.0,6.5$ (the two youngest isochrones in the top panel) and $\log (t)=7.0,7.5,8.0$, and 8.5 (the bottom panel) in the $M_{W_{r i}}$ vs $\mathrm{H}_{\alpha}$ index plane (here $M_{W_{r i}}$ is the absolute Wesenheit magnitude). Note again the characteristic leftward tip where the two branches of the isochrone meet. This time the branch corresponding to lower-mass stars remains almost flat from $\mathrm{H}_{\alpha}$ index $\sim 0$ to $\mathrm{H}_{\alpha}$ index $\sim 0.1$ and then goes down toward fainter absolute Wesenheit magnitides. Furthermore, the absolute Wesenheit magnitude $M_{W_{r i}}$ in the flat part of the diagram is practically independent of age for $\log (t) \geqslant 7.5$. Hence some knowledge of the cluster age (if $\log (t) \sim 6.0-7.5$ ) or just the knowledge that it is not younger than $\log (t) \leqslant 7.5$ is sufficient for estimating rather accurately the cluster distance. Hereafter we fit the observed $\left(\mathrm{H}_{\alpha}\right.$ index, $\left.W_{r i}\right)$ diagram to a theoretical diagram to simultaneously determine the cluster age and distance. Like in the case of the $\left(\mathrm{H}_{\alpha}\right.$ index, $\left.M_{r}\right)$ diagram we also introduce an extra free parameter to allow for a shift in the $\mathrm{H}_{\alpha}$ index $\left(\Delta \mathrm{H}_{\alpha}\right.$ index $)$ in the sense $\mathrm{H}_{\alpha}$ index (observed) $=\mathrm{H}_{\alpha}$ index (theoretical) $+\Delta \mathrm{H}_{\alpha}$ index to correct for a small inconsistency between the theoretical and observed colours. To illustrate this case, in Fig. 7 we plot the corresponding diagrams for clusters NGC 663 and NGC 884. The observed data points can be seen to follow the curves rather closely despite appreciable extinction variations, which amount to $\Delta E_{B-V}=0.43$ and 0.30 for NGC 663 and NGC 884, respectively Yadav et al. 2001). These diagrams imply the distance estimates of $d=2346$ and 2402 pc for NGC 663 and NGC 884, respectively, which agree quite well with the 2420 and 2414 pc estimate reported in the catalogue by Dias et al. (2002) and Mevnet et al. (1993), respectively, although this
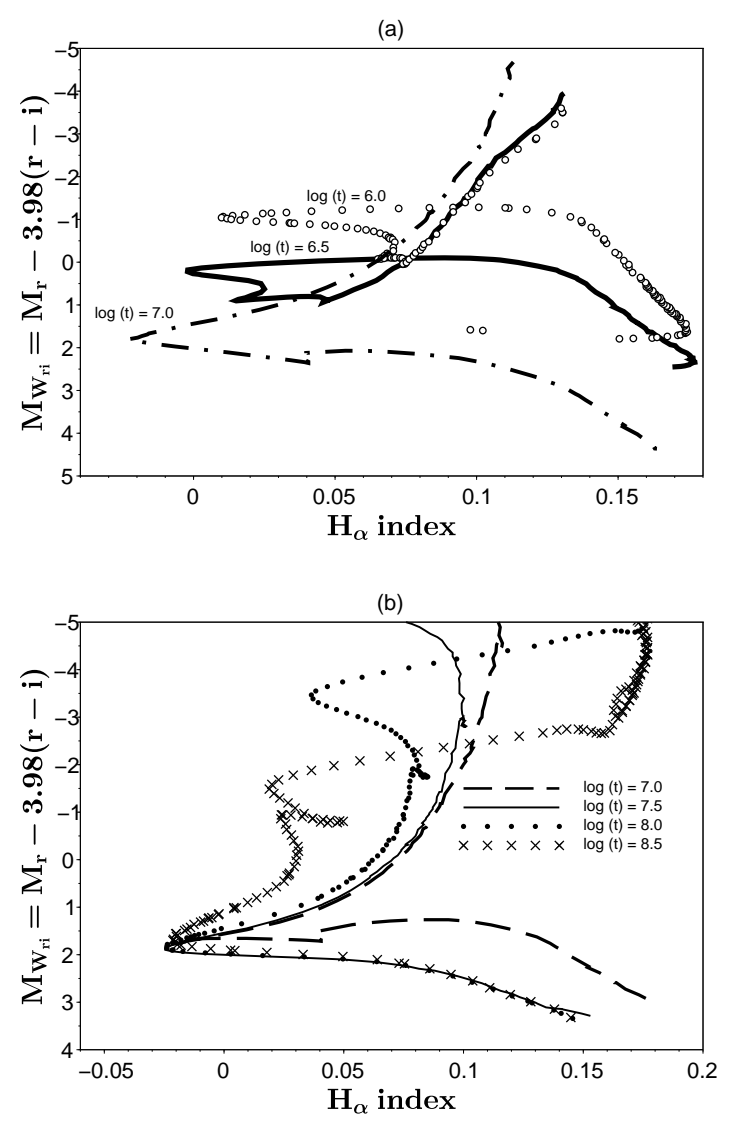

Figure 6. The $\left(\mathrm{H}_{\alpha}\right.$ index, $\left.M_{W_{r i}}\right)$ diagram for isochrones: (a) $\log (t)=6.0,6.5$ and (b) $\log (t)=7.0,7.5,8.0$, and 8.5.

NGC 663 distance estimate is appreciably smaller than the $d=2818$ pc estimate by Paunzen and Netopil (2006).

Finally, it would be good to estimate individual $E_{r-i}=(r-i)-(r-i)_{0}$ extinction values at least for some of the stars in the cluster field. The most evident way is to determine the the $(r-i)_{0}$ intrinsic colour from $\mathrm{H}_{\alpha}$ index using the appropriate isochrone (see Fig. 1). To do this, we need to know the age $(\log (t))$ of the cluster and the systematic offset $\left(\Delta \mathrm{H}_{\alpha}\right.$ index $)$ such that $\mathrm{H}_{\alpha}$ index (theoretical) $=\mathrm{H}_{\alpha}$ index (observed) $-\Delta \mathrm{H}_{\alpha}$ index, both of which can be estimated by fitting the $\left(\mathrm{H}_{\alpha}\right.$-index, $\left.W_{r i}\right)$ diagram (see above). It can be seen from Fig. 1 that the best and most robust estimates of the $(r-i)_{0}$ intrinsic colour and hence the $E_{r-i}=(r-i)-(r-i)_{0}$ colour excess can be obtained for stars on the rising part of the $\left(r-i, \mathrm{H}_{\alpha}\right.$ index $)$ colour-colour diagram with $(r-i)_{0} \sim 0.1-0.4$ and $\mathrm{H}_{\alpha}$ index $\sim 0.0-0.16$ : all isochrones considered are practically indistinguishable throughout this portion of the diagram except for the two youngest ones $(\log (t)=6.0$ and $\log (t)=6.5)$, which deviate from older ones at $(r-i)_{0} \sim 0.1-0.2$ and $\mathrm{H}_{\alpha}$ index $\leqslant 0.05$ (and even these follow closely the overall dependence in the $(r-i)_{0} \sim 0.2-0.4$ and $\mathrm{H}_{\alpha}$ index $\sim 0.05-0.16$ domain $)$. Furthermore, as we show in Section 6 the offsets $\Delta \mathrm{H}_{\alpha}$ index are very similar for all clusters and are, on the average, equal to $<\Delta \mathrm{H}_{\alpha}$ index $>=+0.051 \pm 0.015$, and hence this average value can be used indiscriminately for all clusters without appreciable loss of accuracy. Padova isochrones yield the fol- 

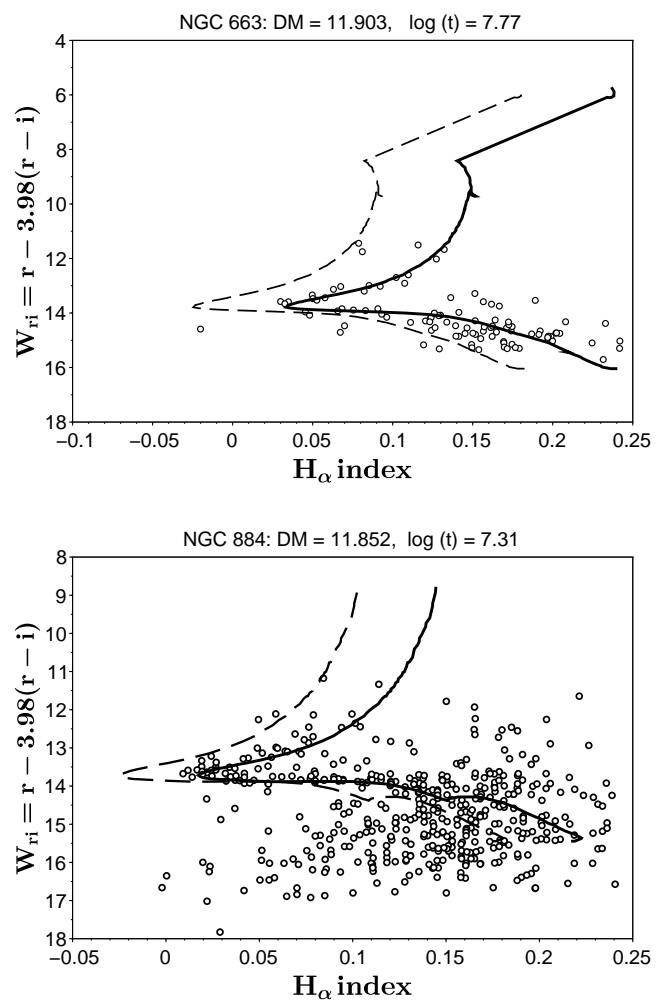

Figure 7. Top panel: the $\left(\mathrm{H}_{\alpha}\right.$ index, $\left.W_{r i}\right)$ "colour-magnitude" diagram for stars within 3 arcmin and from the centre of the NGC 663 cluster with the superimposed $\log (t)=7.77$ isochrone shifted by $11.903 \mathrm{mag}$ along the vertical axis (the dashed curve) and the same isochrone shifted by $11.903 \mathrm{mag}$ (the true distance modulus) along the vertical axis and by $\Delta \mathrm{H}_{\alpha}$ index $=+0.058$ along the horizontal axis (the solid curve). Bottom panel: the $\left(\mathrm{H}_{\alpha}\right.$ index, $\left.W_{r i}\right)$ "colour-magnitude" diagram for stars within 5 arcmin and from the centre of the NGC 884 cluster with the superimposed $\log (t)=7.31$ isochrone shifted by $11.852 \mathrm{mag}$ along the vertical axis (the dashed curve) and the same isochrone shifted by $11.852 \mathrm{mag}$ (the true distance modulus) along the vertical axis and by $\Delta \mathrm{H}_{\alpha}$ index $=+0.042$ along the horizontal axis (the solid curve).

lowing common $(r-i)_{0}\left(\mathrm{H}_{\alpha}\right.$ index(observed $\left.)\right)$ calibration for stars with $(r-i)_{0} \sim 0.1-0.45$ and $\mathrm{H}_{\alpha}$ index $\sim 0-0.17$ and ages in the $\log (t)=7.0-8.5$ interval:

$(r-i)_{0}=+0.099+1.417 x+6.59 x^{2}-86.18 x^{3}+424.0 x^{4}(3)$

Here $x$ is the the estimated "theoretical" $\mathrm{H}_{\alpha}$ index value, which in practice can be computed as

$x=H_{\alpha}$ index $($ observed $)-<\Delta H_{\alpha}$ index $>$,

where we can adopt $<\Delta H_{\alpha}$ index $>=0.051$ (see Section 6 and equation (5) below). Fig. 8 shows this calibration relation along with $\log (t)=6.0-8.0$ isochrones.

To illustrate this method, we compute the color excesses $E_{r-i}=(r-i)-(r-i)_{0}$ of individual stars in the clusters NGC 663 and NGC 884 known for their variable extinction (see above) and show the corresponding histograms in Fig.9.

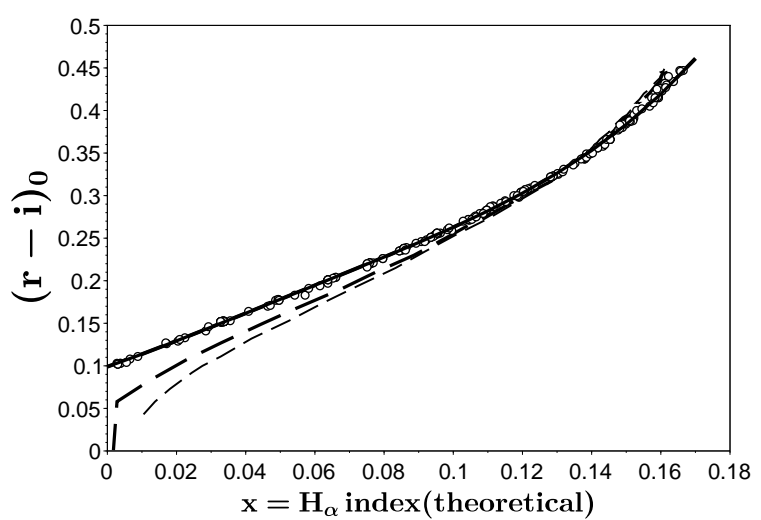

Figure 8. The $\mathrm{H}_{\alpha}$ index(theoretical)- $(r-i)_{0}$ diagram in the $\mathrm{H}_{\alpha}$ index (theoretical $)=0.0-0.16$ and $(r-i)_{0}=0.0-0.45$ domain with $\log (t)=7.0-8.5$ isochrones (the circles) and calibration relation (3) (the solid line). The thin and thick dashed lines show the $\log (t)=6.0$ and $\log (t)=6.5$ isochrones.
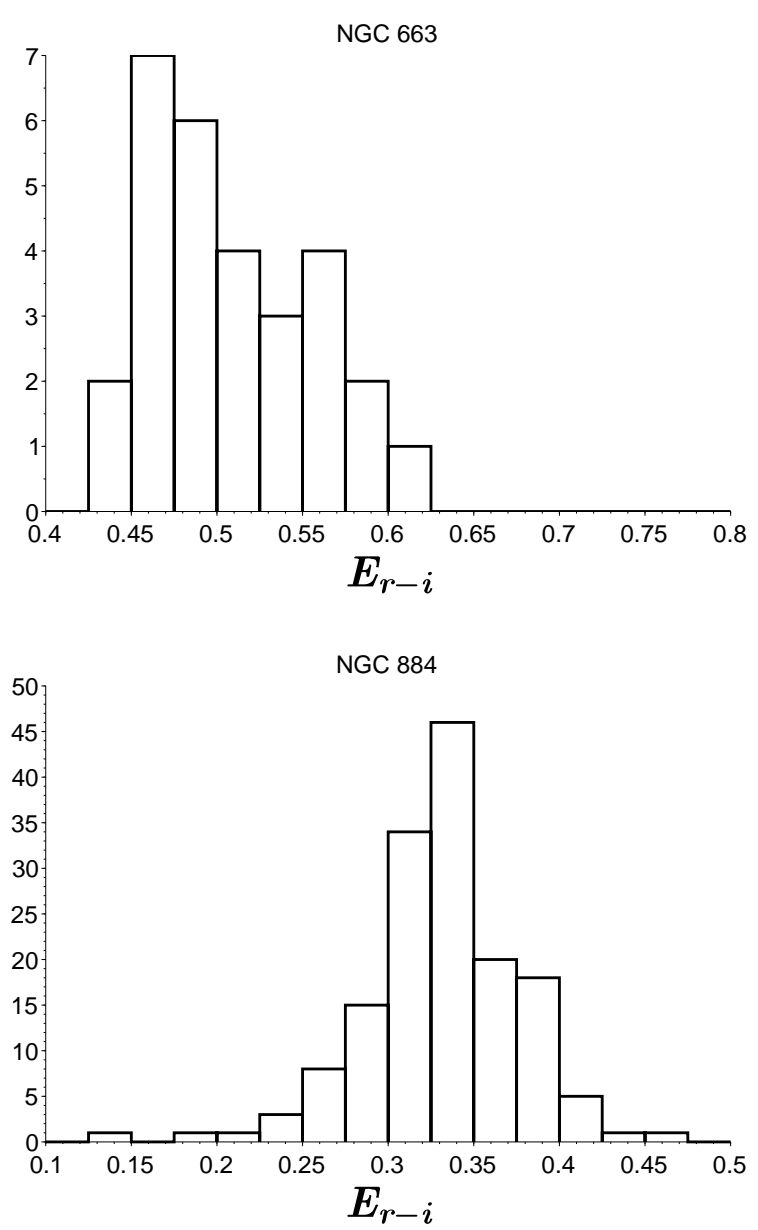

Figure 9. The distribution colour excess values $E_{r-i}=(r-i)-$ $(r-i)_{0}$ computed using calibration (3) for individual stars in the clusters NGC 663 (the top panel) and NGC 884 (the bottom panel). 


\subsection{Application of the maximum-likelihood technique of Naylor and Jefrries and error estimates}

Although the two diagnostic diagrams just described $[(r-$ $i, \mathrm{H}_{\alpha}$ index $)$ and $\left(\mathrm{H}_{\alpha}\right.$ index, $\left.\left.M_{r}\right)\right]$ allow the average color excess and distance modulus of a cluster to be determined rather accurately "by eye", such "manual" estimates have an important drawback: errors of the parameters derived from "eye" estimates are difficult to assess. That is why we refine our initial estimates by applying the formal maximum-likelihood based method proposed by Navlor \& Jeffries (2006), Jeffries et al. (2007), and Mayne et al. (2007), which we adapt for the case of IPHAS magnitudes and colours.

The idea of this method is to use a generalized $\chi^{2}$ statistic proposed by the above authors, which they call the $\tau^{2}$ statistic and which includes uncertainties in two dimensions (e.g., color and magnitude or two different colors) and models with a two-dimensional distribution rather than just single isochronal lines. The $\tau^{2}$ value for each star measures the likelihood for the particular model based on a certain isochrone and shifted by a certain distance modulus, extinction, and $H_{\alpha}$-index offset values to generate a star at the observed position in the diagram taking into account the effects of binary population and photometric errors, and the $\tau^{2}$ value for the cluster is computed by summing up the corresponding values for individual stars. The best-fit model (parametrized by reddening, age, distance and $H_{\alpha}$-index offset or, generally, by a subset of these parameters) is supposed to be the one with minimum $\tau^{2}$ and the corresponding parameter values are adopted as the final estimates. To compute the errors of the inferred parameters, we use the simple bootstrap method with replacement (Efron 1979; Hastie et al. 2009) as described in Section 4.3 of Andrae (2010).

\section{INVESTIGATION OF "STANDARD" CLUSTERS}

We validated our technique of the determination of the three basic cluster parameters colour excess and distance by applying it to "standard" clusters, i.e., to the clusters whose parameters were reliably determined in earlier studies. To this end we compare the cluster parameters colour excess $E_{B-V}$, distance, and age that we determined from IPHAS $\mathrm{H}_{\alpha}$ ri photometry with the corresponding estimates earlier obtained by different authors. We used the relation $E_{B-V}=E_{r-i} / 0.673$ to convert the colour excess $E_{r-i}$ into $E_{B-V}$, and computed the true distance modules by formula $(m-M)_{0}=(m-M)_{r}-3.98 E_{r-i}$. The coefficients 0.673 and 3.98 are computed in accordance with the reddening law of Cardelli et al. (1989) and adopted from the table of photometric system parameters provided by Bressan et al. (2012) along with the isochrones. We constructed the $\left(r-i, \mathrm{H}_{\alpha}\right.$ index $),\left(\mathrm{H}_{\alpha}\right.$ index, $\left.r\right)$, and $\left(\mathrm{H}_{\alpha}\right.$ index, $\left.W_{r i}\right)$ diagrams for all stars inside the radii 3,5 , and 7 arcmin from the cluster centre depending on the angular size of the cluster.

We first investigated the clusters whose distance, age, and $E_{B-V}$ colour excess were published by Paunzen and Netopil (2006). The above authors compiled the list of 72 open clusters whose parameters have been determined repeatedly by different researchers, and for which standard, relatively accurate (in statistical sense) values of physical parameters have been determined. We selected only those clusters among the 72 clusters analysed by Paunzen and Netopil (2006) that are located in the area covered by IPHAS and are no older than $\log (t)=8.5$ according to Paunzen and Netopil (2006). We found few such clusters and therefore we also added open clusters studied by Mevnet et al. (1993), who also determined bona fide parameter estimates for a number of clusters and derived some calibrations for estimating the cluster age from the blue and red MS turnoff points (here the blue and red MS turnoff points are terms introduced by the above authors and graphically explained in their fig. 3). Meynet et al. (1993) studied only the clusters that required no membership determination for individual stars because photoelectric photometry was already performed mostly for cluster members. We converted the apparent distance moduli published by Meynet et al. (1993) into distances using normal extinction law $A_{V}=3.1$ $E_{B-V}$. Given that the most accurate estimates of $E_{B-V}$ colour excesses are those inferred from $(U-B, B-V)$ colourcolour diagrams, we added to our sample the clusters with parameter estimates adopted from Phelps and Janes (1994); Keller et al. (2005), and Lata et al. (2014). The above authors first determined the $E_{B-V}$ colour excess by fitting cluster MS on the $(U-B, B-V)$ diagram to the standard ZAMS of Schmidt-Kaler (1982) or to the youngest isochrones of Girardi et al. (2002), and then determined the distance modulus and age from the colour-magnitude diagrams.

We proceed as follows.

- For each cluster we first estimate its average colour excess $E_{r-i}$ and $\Delta \mathrm{H}_{\alpha}$ index offset by fitting the $\log (t)=7.2$ isochrone (with the age approximatively midway between our age interval boundaries $(\log (t)=6.0$ and $\log (t)=8.5)$ to the $\left(r-i, \mathrm{H}_{\alpha}\right.$ index) diagram based on IPHAS data (see Fig. 2).

- We then use this initial $E_{r-i}$ estimate to fit the appropriate isochrone to the $(r-i)-r$ diagram based on IPHAS and transformed APASS data (the latter are used for stars that are too bright and hence saturated in the IPHAS frames) and infer a preliminary age estimate $\left(\log \left(t_{0}\right)-\right.$ see Fig. 3).

- We then return to the first step and refine the colour excess $E_{r-i}$ and $\Delta \mathrm{H}_{\alpha}$ index offset by fitting the $\left(r-i, \mathrm{H}_{\alpha}\right.$ index) diagram to the isochrone with the preliminary age estimate (instead of $\log (t)=7.2$ ).

- We now use the refined $E_{r-i}$ estimate to fit the appropriate isochrone to the $(r-i)-r$ diagram and determine the final age $\left(\log \left(\mathrm{t}_{1}\right)\right)$ and apparent distance modulus $\left(D M_{r}\right)$ estimates and compute the first distance $\left(D_{1}\right)$ estimates by dereddening the inferred apparent distance modulus.

- We then fit the $\left(\mathrm{H}_{\alpha}\right.$ index, $\left.r\right)$ diagram for cluster stars with the $\left(\mathrm{H}_{\alpha}\right.$ index, $\left.M_{r}\right)$ isochrone with fixed $\log \left(\mathrm{t}_{1}\right)$ (see Fig. 5) and determine another distance $\left(\mathrm{D}_{2}\right)$ and another $\Delta \mathrm{H}_{\alpha}$ index offset estimate.

- We finally fit an appropriate isochrone to the reddeningfree $\left(\mathrm{H}_{\alpha}\right.$ index, $\left.W_{r i}\right)$ diagram (see Fig. 7) with the true distance modulus, age, and $\Delta \mathrm{H}_{\alpha}$ index offset treated as free parameters to determine the third distance $\left(D_{3}\right)$ and 
the third $\Delta \mathrm{H}_{\alpha}$ index offset estimates and the second age estimate $\left(\log \left(t_{2}\right)\right)$ for the cluster.

- Finally, we determine the monochromatic extinction $A_{0}$ at $5495 \AA$ from the $3 \mathrm{D}$ map of Sale et al. (2014) and the $A_{V}-A_{0}$ difference, where $A_{V}=3.1 E_{B-V}$ (we compute $\left.E_{B-V}=E_{r-i} / 0.673\right)$ is our total V-band extinction estimate.

\section{RESULTS}

The results for all 18 "standard" clusters are summarized in Table 2. Here column 1 gives the cluster name; column 2, our final $E_{B-V}$ colour excess estimate $\left(E_{B-V}=E_{r-i} / 0.673\right)$ (at the top of the cell) and published $E_{B-V}$ estimates (at the bottom of the cell); column 3 gives our three distance estimates based on three photometric diagrams (indicated in the parentheses) at the top of the cell followed by published distance estimates at the bottom of the cell; column 4 lists our two age estimates based on two photometric diagrams (indicated in the parentheses) at the top of the cell followed by published age estimates at the bottom of the cell; column 5 gives the corresponding references to the published colour excess, distance, and age values listed in columns 2-4; column 6 gives the monochromatic extinction $A_{0}$ at $5495 \AA$ from the 3D map of Sale et al. (2014), and column 7, the $A_{V}-A_{0}$ difference.

For comparison, we also list in Table 2 the cluster parameters from the recent version of DAML02 open cluster catalog by Dias et al. (2002) (columns 2-4, the bottom part of the cells) except for the clusters for which DAML02 adopted the color excess, distance, and age from the studies cited above.

Our extinction and distance estimates agree well with those reported in most of the standard cluster studies - Paunzen and Netopil (2006); Mevnet et al. (1993); Phelps and Janes (1994); Lata et al. (2014); Keller et al. (2005):

$$
\begin{gathered}
<E_{B-V}(P u b l)-E_{B-V}>=-0.005 \pm 0.012, \\
\sigma\left(E_{B-V}(P u b l)-E_{B-V}\right)=0.061
\end{gathered}
$$

where $E_{B-V}$ and $E_{B-V}(P u b l)$ are our inferred extinction values and the original published estimates of the above authors, respectively (see Table 3 and Fig. 10).

On the other hand, our extinction values deviate appreciably from those implied by the $3 \mathrm{D}$ map of Sale et al. (2014): we have in the case of the $A_{V}-A_{0}$ difference, where $A_{V}$ is our total V-band extinction estimate:

$<A_{V}-A_{0}>=+0.251 \pm 0.018, \sigma\left(A_{V}-A_{0}\right)=0.077$,

or, in terms of colour excesses

$$
\begin{gathered}
<E_{B-V}-\left(A_{0} / 3.1\right)>=+0.081 \pm 0.006 \\
\sigma\left(E_{B-V}-\left(A_{0} / 3.1\right)\right)=0.025
\end{gathered}
$$

(see Table 3 and Fig. 10). This difference may be due to the limits imposed by the finite sampling of the extinction map of Sale et al. (2014) mentioned above: here we adopted the
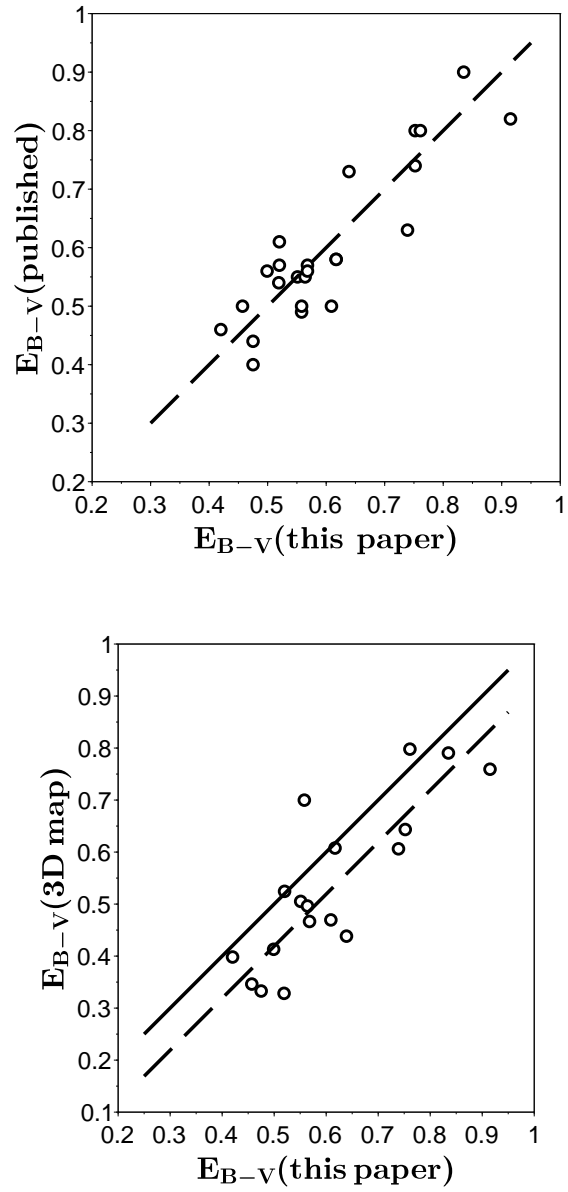

Figure 10. Top panel: comparison of our $E_{B-V}$ colour excess estimates for "standard" clusters with published values. The dashed line corresponds to $E_{B-V}$ (published) $=E_{B-V}$ (this paper). Bottom panel: comparison of our $E_{B-V}$ colour excess estimates for "standard" clusters with those implied by the 3D extinction map by Sale et al. (2014). The dashed line corresponds to $E_{B-V}(3 \mathrm{D}$ map) $=E_{B-V}$ (this paper)-0.081 relation.

$A_{0}$ values corresponding to sky locations $5-7$ arcmin from the cluster centre in most of the cases. The systematically higher values of our extinction estimates may be due to internal extinction within the cluster.

Our distance estimates based on the $(r-i, r),\left(\mathrm{H}_{\alpha}\right.$ index, $r)$, and $\left(\mathrm{H}_{\alpha}\right.$ index, $\left.W_{r i}\right)$ diagrams agree well with each other:

$$
<D\left(r, H_{\alpha} \text { index }\right) / D(r-i, r)>=0.980 \pm 0.009,
$$

$$
\sigma\left(D\left(r, H_{\alpha} \text { index }\right) / D(r-i, r)\right)=0.037,
$$

and

$$
<\left(D\left(W_{r i}, H_{\alpha} \text { index }\right) / D(r-i, r)>=1.000 \pm 0.008\right.
$$

$$
\sigma\left(\left(D\left(W_{r i}, H_{\alpha} \text { index }\right) / D(r-i, r)\right)=0.035 .\right.
$$

They also agree well with earlier published values except for the NGC6834 cluster for which we obtain a significantly greater distance $(3115 \pm 63,3115 \pm 120$, and $2950 \pm 59$ 


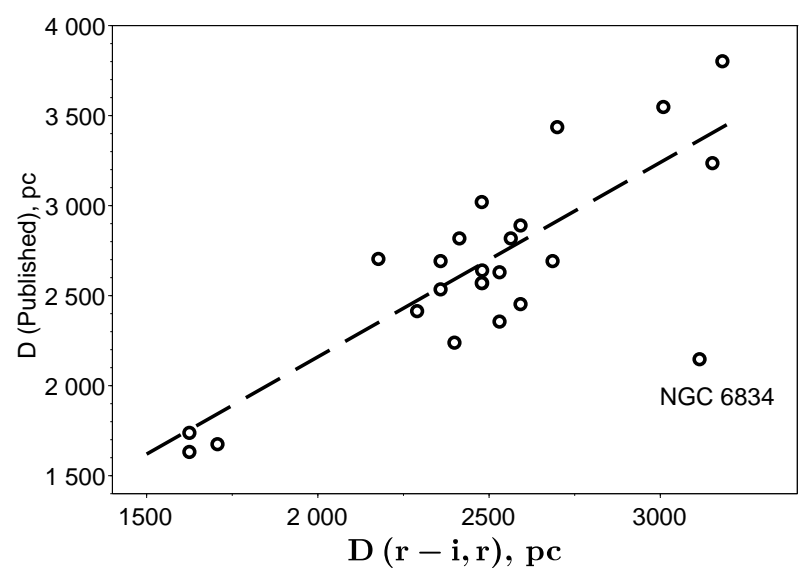

Figure 11. Comparison of our distance estimates $D(r-i, r)$ with published values. The dashed line is the $D($ Published $)=1.080 D(r-i, r)$ relation inferred from all clusters except NGC 6834.

based on $(r-i, r),\left(\mathrm{H}_{\alpha}\right.$ index, $\left.r\right)$, and $\left(\mathrm{H}_{\alpha}\right.$ index, $\left.W_{r i}\right)$ diagrams, respectively) than inferred by Paunzen and Netopil (2006) $(2147 \pm 59)$ (see Fig. 11):

$$
\begin{gathered}
<D(\text { Published }) / D(r-i, r)>=1.080 \pm 0.020, \\
\sigma(D(\text { Published }) / D(r-i, r))=0.098, \\
<D(\text { Published }) / D\left(r, H_{\alpha} \text { index }\right)>=1.105 \pm 0.026, \\
\sigma\left(D(\text { Published }) / D\left(r, H_{\alpha} \text { index }\right)\right)=0.123,
\end{gathered}
$$

and

$$
<D(\text { Published }) / D\left(W_{r i}, H_{\alpha} \text { index }\right)>=1.075 \pm 0.020
$$

$$
\sigma\left(D(\text { Published }) / D\left(W_{\text {ri }}, H_{\alpha} \text { index }\right)\right)=0.094,
$$

where $D($ Published $)$ are published distance estimates adopted from Paunzen and Netopil (2006); Meynet et al. (1993); Phelps and Janes (1994); Lata et al. (2014); Keller et al. (2005) and $D(r-i, i), D\left(r, H_{\alpha}\right.$ index $)$, and $D\left(W_{r i}, H_{\alpha}\right.$ index $)$ are our distance estimates based on the $(r-i, i),\left(r, H_{\alpha}\right.$ index $)$, and $D\left(W_{r i}, H_{\alpha}\right.$ index $)$ diagrams, respectively (see Table 3 ).

The overall good agreement of our inferred colour excesses and distances with earlier estimates suggests that the technique presented in this work can be used to estimate the two above parameters for those clusters that do not have observations in the $U$ band.

However, our age estimates based on the $(r-i, i)$ and $\left(W_{r i}, H_{\alpha}\right.$ index $)$ diagrams do not agree so well with published values:

$$
<\log (t)(\text { Published })-\log (t)(r-i, r)>=+0.33 \pm 0.06,
$$

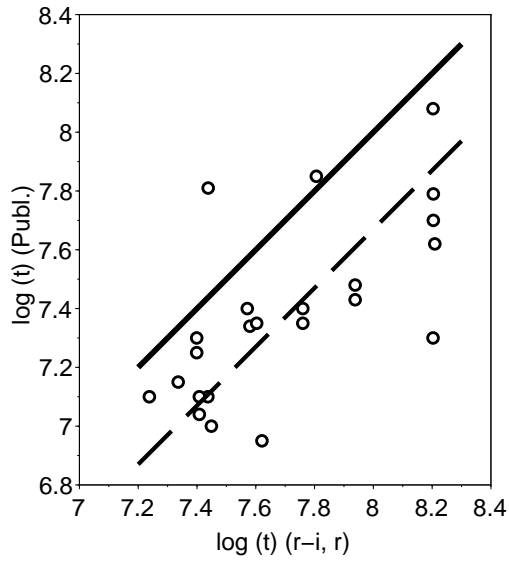

Figure 12. Comparison of our age estimates log $(t(r-$ $i, r)$ ) with published values. The solid and dashed lines show the identity $(\log (\mathrm{t}($ Published $))=\log (\mathrm{t}(r-i, r)))$ and $\log (\mathrm{t}($ Published $))=\log (\mathrm{t}(r-i, r))-0.33$ relations, respectively.

Table 1. Intrinsic $r-i$ colour of the $\mathrm{H}_{\alpha}$ index minimum feature as a function of age and metallicity.

\begin{tabular}{rrrr}
\hline & \multicolumn{3}{c}{ r-i at minimum $\mathrm{H}_{\alpha}$ index } \\
Age, $\log (t)$ & $\mathrm{Z}=0.0048$ & $\mathrm{Z}=0.0152$ & $\mathrm{Z}=0.0481$ \\
& {$[\mathrm{Fe} / \mathrm{H}]=-0.5$} & {$[\mathrm{Fe} / \mathrm{H}]=0.0$} & {$[\mathrm{Fe} / \mathrm{H}]=+0.5$} \\
\hline 6.0 & 0.032 & 0.027 & 0.032 \\
6.5 & 0.024 & 0.024 & 0.027 \\
7.0 & 0.020 & 0.020 & 0.021 \\
7.5 & 0.022 & 0.024 & 0.026 \\
8.0 & 0.022 & 0.024 & 0.027 \\
8.5 & 0.026 & 0.031 & 0.032 \\
\hline Mean & 0.028 & 0.025 & 0.026 \\
& \pm 0.004 & \pm 0.004 & \pm 0.004 \\
\hline Overall mean & \multicolumn{3}{c}{$0.026 \pm 0.004$} \\
\hline
\end{tabular}

and

$$
\begin{gathered}
<\log (t)(\text { Published })-\log (t)\left(W_{r i}, H_{\alpha} \text { index }\right)>=+0.46 \pm 0.07, \\
\sigma\left(\log (t)(\text { Published })-\log (t)\left(W_{r i}, H_{\alpha} \text { index }\right)\right)=0.36,
\end{gathered}
$$

which is to be expected given the saturation of bright stars in IPHAS images. As expected, the results based on the $(r-i, r)$ diagram are slightly better than those based on the $\left(W_{r i}, H_{\alpha}\right.$ index) diagram because the former includes the data adopted from APASS for relatively bright evolved stars (see Table 4 and Fig. 12). 
Table 2: Comparison of parameter estimates for "standard" open clusters.

\begin{tabular}{|c|c|c|c|c|c|c|}
\hline Cluster & $E_{B-V}$ & $\mathrm{~d}^{*}, \mathrm{pc}$ & $\log (t)^{*}$ & reference $^{* *}$ & $\mathrm{~A}_{0}$ & $\mathrm{~A}_{v}-\mathrm{A}_{0}\left({ }^{* * *}\right)$ \\
\hline Be 7 & $\begin{array}{r}0.752 \\
\pm 0.009\end{array}$ & $\begin{array}{l}2480 \pm 87(r-i, r) \\
2513 \pm 42\left(\mathrm{H}_{\alpha} \text { index }, r\right) \\
2484 \pm 140\left(\mathrm{H}_{\alpha} \text { index }, W\right) \\
2570 \\
2640\end{array}$ & $\begin{array}{l}7.44 \pm 0.16(r-i, r) \\
7.41 \pm 0.24\left(\mathrm{H}_{\alpha} \text { index, } W\right) \\
6.60 \\
7.1\end{array}$ & $\begin{array}{l}\text { This } \\
\text { paper } \\
\\
\text { PJ94 } \\
\text { L14 }\end{array}$ & $\begin{array}{r}1.99 \\
\pm 0.04\end{array}$ & +0.34 \\
\hline Be 62 & $\begin{array}{r}0.915 \\
\pm 0.015\end{array}$ & $\begin{array}{l}2177 \pm 34(r-i, r) \\
2513 \pm 42\left(\mathrm{H}_{\alpha} \text { index }, r\right) \\
2250 \pm 45\left(\mathrm{H}_{\alpha} \text { index }, W\right) \\
2704 \\
2320\end{array}$ & $\begin{array}{l}7.45 \pm 0.10(r-i, r) \\
7.91 \pm 0.18\left(\mathrm{H}_{\alpha} \text { index, } W\right) \\
7.00 \\
7.2\end{array}$ & $\begin{array}{l}\text { This } \\
\text { paper } \\
\text { PJ94 } \\
\text { DAML02 }\end{array}$ & $\begin{array}{r}2.35 \\
\pm 0.04\end{array}$ & +0.49 \\
\hline King 12 & $\begin{array}{r}0.618 \\
\pm 0.007 \\
\\
\\
0.58 \\
\pm 0.03 \\
0.58\end{array}$ & $\begin{array}{l}2592 \pm 46(r-i, r) \\
2339 \pm 64\left(\mathrm{H}_{\alpha} \text { index, } r\right) \\
2595 \pm 87\left(\mathrm{H}_{\alpha} \text { index, } W\right) \\
2453 \pm 120 \\
2890\end{array}$ & $\begin{array}{l}7.41 \pm 0.03(r-i, r) \\
7.22 \pm 0.04\left(\mathrm{H}_{\alpha} \text { index, } W\right) \\
7.04 \\
7.1\end{array}$ & $\begin{array}{l}\text { This } \\
\text { paper } \\
\text { PN06 } \\
\text { L14 }\end{array}$ & $\begin{array}{r}1.88 \\
\pm 0.06\end{array}$ & +0.04 \\
\hline NGC 103 & $\begin{array}{r}0.551 \\
\pm 0.010 \\
\\
\\
0.55 \\
0.406 \\
\end{array}$ & $\begin{array}{l}3181 \pm 38(r-i, r) \\
3307 \pm 110\left(\mathrm{H}_{\alpha} \text { index, } r\right) \\
3199 \pm 73\left(\mathrm{H}_{\alpha} \text { index, } W\right) \\
3802 \\
3026\end{array}$ & $\begin{array}{l}8.20 \pm 0.06(r-i, r) \\
8.26 \pm 0.10\left(\mathrm{H}_{\alpha} \text { index, } W\right) \\
7.30 \\
8.126\end{array}$ & $\begin{array}{l}\text { This } \\
\text { paper } \\
\text { PJ94 } \\
\text { DAML02 }\end{array}$ & $\begin{array}{r}1.57 \\
\pm 0.04\end{array}$ & +0.14 \\
\hline NGC 129 & $\begin{array}{r}0.569 \\
\pm 0.018 \\
\\
\\
0.56 \\
\pm 0.03 \\
0.57 \\
0.548\end{array}$ & $\begin{array}{l}1625 \pm 14(r-i, r) \\
1605 \pm 24\left(\mathrm{H}_{\alpha} \text { index, } r\right) \\
1690 \pm 20\left(\mathrm{H}_{\alpha} \text { index, } W\right) \\
1632 \pm 56 \\
1738 \\
1625\end{array}$ & $\begin{array}{l}8.20 \pm 0.04(r-i, r) \\
8.30 \pm 0.01\left(\mathrm{H}_{\alpha} \text { index, } W\right) \\
7.79 \\
7.70 \\
7.886\end{array}$ & $\begin{array}{l}\text { This } \\
\text { paper } \\
\text { PN06 } \\
\text { PJ94 } \\
\text { DAML02 }\end{array}$ & $\begin{array}{r}1.45 \\
\pm 0.04\end{array}$ & +0.31 \\
\hline NGC 436 & $\begin{array}{r}0.458 \\
\pm 0.006 \\
\\
\\
0.50 \\
0.460\end{array}$ & $\begin{array}{l}3152 \pm 34(r-i, r) \\
3089 \pm 60\left(\mathrm{H}_{\alpha} \text { index, } r\right) \\
3070 \pm 26\left(\mathrm{H}_{\alpha} \text { index, } W\right) \\
3236 \\
3014\end{array}$ & $\begin{array}{l}8.21 \pm 0.04(r-i, r) \\
8.10 \pm 0.08\left(\mathrm{H}_{\alpha} \text { index, } W\right) \\
7.62 \\
7.926\end{array}$ & $\begin{array}{l}\text { This } \\
\text { paper } \\
\text { PN06 } \\
\text { DAML02 }\end{array}$ & $\begin{array}{r}1.07 \\
\pm 0.04\end{array}$ & +0.35 \\
\hline NGC 457 & $\begin{array}{r}0.559 \\
\pm 0.006 \\
\\
0.50 \\
0.49 \\
0.472\end{array}$ & $\begin{array}{l}2479 \pm 39(r-i, r) \\
2331 \pm 26\left(\mathrm{H}_{\alpha} \text { index, } r\right) \\
2476 \pm 30\left(\mathrm{H}_{\alpha} \text { index, } W\right) \\
2570 \\
3020 \\
2429\end{array}$ & $\begin{array}{l}7.40 \pm 0.07(r-i, r) \\
8.10 \pm 0.09\left(\mathrm{H}_{\alpha} \text { index, } W\right) \\
7.25 \\
7.30 \\
7.324\end{array}$ & $\begin{array}{l}\text { This } \\
\text { paper } \\
\text { M93 } \\
\text { PJ94 } \\
\text { DAML02 }\end{array}$ & $\begin{array}{r}1.43 \\
\pm 0.01\end{array}$ & +0.28 \\
\hline NGC 581 & $\begin{array}{r}0.475 \\
\pm 0.006 \\
\\
\\
0.40 \\
0.44 \\
0.382\end{array}$ & $\begin{array}{l}2358 \pm 20(r-i, r) \\
2207 \pm 150\left(\mathrm{H}_{\alpha} \text { index }, r\right) \\
2427 \pm 150\left(\mathrm{H}_{\alpha} \text { index, } W\right) \\
2535 \\
2692 \\
2194\end{array}$ & $\begin{array}{l}7.76 \pm 0.06(r-i, r) \\
8.22 \pm 0.14\left(\mathrm{H}_{\alpha} \text { index, } W\right) \\
7.40 \\
7.35 \\
7.336\end{array}$ & $\begin{array}{l}\text { This } \\
\text { paper } \\
\text { M93 } \\
\text { PJ96 } \\
\text { DAML02 }\end{array}$ & $\begin{array}{r}1.03 \\
\pm 0.03\end{array}$ & +0.44 \\
\hline
\end{tabular}

* The colour-magnitude diagram used to compute $d$ and log $(t)$ is indicated in the parentheses.

** PN06: Paunzen and Netopil (2006); L14: Lata et al. (2014); DAML02: Dias et al. (2002);

M93: Mevnet et al. (1993); PJ94: Phelps and Janes (1994); K05: Keller et al. (2005).

*** $\mathrm{A}_{v}=3.1 E_{B-V}$

Continued on Next Page. . 
Investigation of open clusters based on IPHAS and APASS survey data

\begin{tabular}{|c|c|c|c|c|c|c|}
\hline Cluster & $E_{B-V}$ & $\mathrm{~d}^{*}, \mathrm{pc}$ & $\log (t)^{*}$ & reference $^{* *}$ & $\overline{A_{0}}$ & $\mathrm{~A}_{v}-\mathrm{A}_{0}\left({ }^{* * *}\right)$ \\
\hline NGC 654 & $\begin{array}{r}0.835 \\
\pm 0.013\end{array}$ & $\begin{array}{l}2685 \pm 75(r-i, r) \\
2507 \pm 54\left(\mathrm{H}_{\alpha} \text { index, } r\right) \\
2704 \pm 73\left(\mathrm{H}_{\alpha} \text { index, } W\right) \\
2692 \\
2410\end{array}$ & $\begin{array}{l}7.57 \pm 0.14(r-i, r) \\
8.10 \pm 0.13\left(\mathrm{H}_{\alpha} \text { index, } W\right) \\
7.40 \\
7.0\end{array}$ & $\begin{array}{l}\text { This } \\
\text { paper } \\
\\
\text { PJ96 } \\
\text { DAML02 }\end{array}$ & $\begin{array}{r}2.45 \\
\pm 0.05\end{array}$ & +0.14 \\
\hline NGC 659 & $\begin{array}{r}0.738 \\
\pm 0.018 \\
\\
0.63 \\
0.652\end{array}$ & $\begin{array}{l}2699 \pm 94(r-i, r) \\
2699 \pm 94\left(\mathrm{H}_{\alpha} \text { index, } r\right) \\
2767 \pm 42\left(\mathrm{H}_{\alpha} \text { index }, W\right) \\
3436 \\
1938\end{array}$ & $\begin{array}{l}7.58 \pm 0.06(r-i, r) \\
7.43 \pm 0.28\left(\mathrm{H}_{\alpha} \text { index, } W\right) \\
7.34 \\
7.548\end{array}$ & $\begin{array}{l}\text { This } \\
\text { paper } \\
\text { PJ96 } \\
\text { DAML02 }\end{array}$ & $\begin{array}{r}1.88 \\
\pm 0.03\end{array}$ & +0.41 \\
\hline NGC 663 & $\begin{array}{r}0.761 \\
\pm 0.015\end{array}$ & $\begin{array}{l}2413 \pm 73(r-i, r) \\
2408 \pm 71\left(\mathrm{H}_{\alpha} \text { index, } r\right) \\
2402 \pm 31\left(\mathrm{H}_{\alpha} \text { index, } W\right) \\
2818 \\
2420\end{array}$ & $\begin{array}{l}7.60 \pm 0.08(r-i, r) \\
7.77 \pm 0.12\left(\mathrm{H}_{\alpha} \text { index }, W\right) \\
7.35 \\
7.4\end{array}$ & $\begin{array}{l}\text { This } \\
\text { paper } \\
\text { PJ06 } \\
\text { DAML02 }\end{array}$ & $\begin{array}{r}2.48 \\
\pm 0.09\end{array}$ & -0.12 \\
\hline NGC 869 & $\begin{array}{r}0.54 \\
0.575\end{array}$ & $\begin{array}{l}2399 \pm 66(r-i, r) \\
2297 \pm 26\left(\mathrm{H}_{\alpha} \text { index, } r\right) \\
2391 \pm 60\left(\mathrm{H}_{\alpha} \text { index, } W\right) \\
2239 \\
2079\end{array}$ & $\begin{array}{l}7.24 \pm 0.04(r-i, r) \\
7.28 \pm 0.06\left(\mathrm{H}_{\alpha} \text { index, } W\right) \\
7.1 \\
7.069\end{array}$ & $\begin{array}{l}\text { This } \\
\text { paper } \\
\text { K05 } \\
\text { DAML02 }\end{array}$ & $\begin{array}{r}1.02 \\
\pm 0.02\end{array}$ & +0.59 \\
\hline NGC 884 & $\begin{array}{r}0.499 \\
\pm 0.009 \\
\\
\\
0.56 \\
0.56\end{array}$ & $\begin{array}{l}2290 \pm 28(r-i, r) \\
2188 \pm 29\left(\mathrm{H}_{\alpha} \text { index, } r\right) \\
2346 \pm 25\left(\mathrm{H}_{\alpha} \text { index, } W\right) \\
2414 \\
2940\end{array}$ & $\begin{array}{l}7.34 \pm 0.12(r-i, r) \\
7.31 \pm 0.09\left(\mathrm{H}_{\alpha} \text { index, } W\right) \\
7.15 \\
7.1\end{array}$ & $\begin{array}{l}\text { This } \\
\text { paper } \\
\text { M93 } \\
\text { DAML02 }\end{array}$ & $\begin{array}{r}1.28 \\
\pm 0.01\end{array}$ & +0.27 \\
\hline NGC 6834 & $\begin{array}{r}0.639 \\
\pm 0.012 \\
\\
0.73 \\
\pm 0.03 \\
\end{array}$ & $\begin{array}{l}3115 \pm 63(r-i, r) \\
3115 \pm 120\left(\mathrm{H}_{\alpha} \text { index, } r\right) \\
2950 \pm 36\left(\mathrm{H}_{\alpha} \text { index }, W\right) \\
2147 \pm 59\end{array}$ & $\begin{array}{l}7.44 \pm 0.12(r-i, r) \\
7.91 \pm 0.10\left(\mathrm{H}_{\alpha} \text { index, } W\right) \\
7.81\end{array}$ & $\begin{array}{l}\text { This } \\
\text { paper } \\
\text { PN06 }\end{array}$ & $\begin{array}{r}1.36 \\
\pm 0.05\end{array}$ & +0.62 \\
\hline NGC 6871 & $\begin{array}{r}0.421 \\
\pm 0.007 \\
\\
\\
0.46 \\
\pm 0.03\end{array}$ & $\begin{array}{l}1707 \pm 43(r-i, r) \\
1617 \pm 73\left(\mathrm{H}_{\alpha} \text { index, } r\right) \\
1615 \pm 41\left(\mathrm{H}_{\alpha} \text { index, } W\right) \\
1675 \pm 130\end{array}$ & $\begin{array}{l}7.62 \pm 0.25(r-i, r) \\
8.02 \pm 0.04\left(\mathrm{H}_{\alpha} \text { index, } W\right) \\
6.95\end{array}$ & $\begin{array}{l}\text { This } \\
\text { paper } \\
\text { PN06 }\end{array}$ & $\begin{array}{r}1.24 \\
\pm 0.09\end{array}$ & +0.07 \\
\hline NGC 7790 & $\begin{array}{r}0.565 \\
\pm 0.006 \\
\\
0.55 \\
0.531\end{array}$ & $\begin{array}{l}3006 \pm 90(r-i, r) \\
2793 \pm 72\left(\mathrm{H}_{\alpha} \text { index, } r\right) \\
2858 \pm 44\left(\mathrm{H}_{\alpha} \text { index, } W\right) \\
3548 \\
2944\end{array}$ & $\begin{array}{l}7.81 \pm 0.23(r-i, r) \\
7.86 \pm 0.17\left(\mathrm{H}_{\alpha} \text { index, } W\right) \\
7.85 \\
7.749\end{array}$ & $\begin{array}{l}\text { This } \\
\text { paper } \\
\text { PJ94 } \\
\text { DAML02 }\end{array}$ & $\begin{array}{r}1.54 \\
\pm \quad 0.04\end{array}$ & +0.21 \\
\hline Stock 24 & $\begin{array}{r}0.609 \\
\pm 0.015 \\
\\
0.50\end{array}$ & $\begin{array}{l}2563 \pm 28(r-i, r) \\
2570 \pm 50\left(\mathrm{H}_{\alpha} \text { index, } r\right) \\
2750 \pm 59\left(\mathrm{H}_{\alpha} \text { index, } W\right) \\
2818\end{array}$ & $\begin{array}{l}8.20 \pm 0.02(r-i, r) \\
8.27 \pm 0.03\left(\mathrm{H}_{\alpha} \text { index, } W\right) \\
8.08\end{array}$ & $\begin{array}{l}\text { This } \\
\text { paper } \\
\text { PJ94 }\end{array}$ & $\begin{array}{r}1.46 \\
\pm 0.06\end{array}$ & +0.43 \\
\hline Trumpler 1 & $\begin{array}{r}0.520 \\
\pm 0.082\end{array}$ & $\begin{array}{l}2530 \pm 42(r-i, r) \\
2903 \pm 160\left(\mathrm{H}_{\alpha} \text { index, } r\right) \\
2437 \pm 37\left(\mathrm{H}_{\alpha} \text { index, } W\right)\end{array}$ & $\begin{array}{l}7.94 \pm 0.18(r-i, r) \\
7.43 \pm 0.10\left(\mathrm{H}_{\alpha} \text { index }, W\right)\end{array}$ & $\begin{array}{l}\text { This } \\
\text { paper }\end{array}$ & $\begin{array}{r}1.63 \\
\pm 0.03\end{array}$ & -0.02 \\
\hline
\end{tabular}

* The colour-magnitude diagram used to compute $d$ and $\log (t)$ is indicated in the parentheses.

** PN06: Paunzen and Netopil (2006); L14: Lata et al. (2014); DAML02: Dias et al. (2002);

M93: Mevnet et al. (1993); PJ94: Phelps and Janes (1994); K05: Keller et al. (2005).

*** $\mathrm{A}_{v}=3.1 E_{B-V}$.

Continued on Next Page. . 


\begin{tabular}{|l|r|l|l|l|l|l|}
\hline Cluster & $E_{B-V}$ & $\mathrm{~d}^{*}, \mathrm{pc}$ & $\log (t)^{*}$ & reference** $^{* *}$ & $\mathrm{~A}_{0}$ & $\mathrm{~A}_{v}-\mathrm{A}_{0}\left(^{* * *}\right)$ \\
\hline & 0.57 & $2356 \pm 510$ & 7.48 & & \\
& \pm 0.04 & & & & \\
& 0.61 & 2630 & 7.43 & PJ94 & & \\
\hline
\end{tabular}

* The colour-magnitude diagram used to compute $d$ and $\log (t)$ is indicated in the parentheses.

** PN06: Paunzen and Netopil (2006); L14: Lata et al. (2014); DAML02: Dias et al. (2002); M93: Mevnet et al. (1993);

PJ94: Phelps and Janes (1994); K05: Keller et al. (2005).

*** $\mathrm{A}_{v}=3.1 E_{B-V}$. 
Investigation of open clusters based on IPHAS and APASS survey data

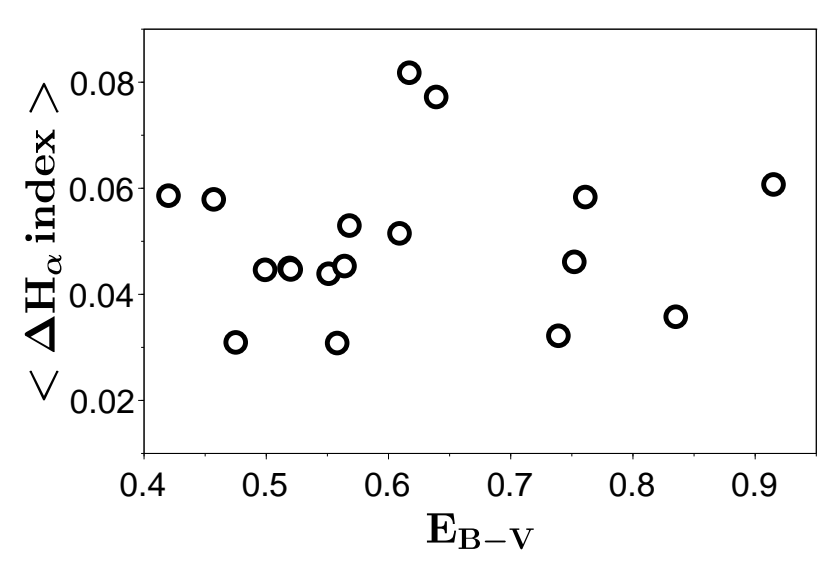

Figure 13. Plot of $\Delta \mathrm{H} \alpha_{\alpha}$-index offset computed as the average of the estimates based on three photometric diagrams versus the $E_{B-V}$ colour excess. The offsets show no trend with colour excess: $<\Delta \mathrm{H}_{\alpha}$-index $>=+0.049( \pm 0.005)+0.007( \pm 0.026)\left(E_{B-V^{-}}-0.5\right)$.

\section{THE SYSTEMATIC $\mathrm{H}_{\alpha}$ INDEX OFFSET}

As we already pointed out above, the observed $\mathrm{H}_{\alpha}$ index values differ systematically from those implied by the corresponding isochrones. Table 5 lists the $\Delta \mathrm{H}_{\alpha}$-index estimates for 18 "standard" clusters based on $\left(r-i, \mathrm{H}_{\alpha}\right.$ index $)$, $\left(\mathrm{H}_{\alpha}\right.$ index, $\left.r\right)$, and $\left(\mathrm{H}_{\alpha}\right.$ index, $\left.W\right)$ diagrams (columns 2 to 4$)$ and the weighted averages of these estimates for each cluster (column 5). All three diagrams yield highly consistent $\Delta \mathrm{H}_{\alpha}$-index values for each cluster with the average difference between the values inferred from the $\left(r-i, H_{\alpha}\right.$ index $)$ and $\left(H_{\alpha}\right.$ index, $\left.r\right)$ diagrams equal to $+0.004 \pm 0.002$ with a scatter of 0.009 and the average difference between the values inferred from the $\left(H_{\alpha}\right.$ index, $\left.r\right)$ and $\left(H_{\alpha}\right.$ index,$\left.W\right)$ diagrams equal to $+0.001 \pm 0.001$ with a scatter of 0.005 .

Furthermore, the scatter of $\Delta \mathrm{H}_{\alpha}$-index values for different clusters is also rather small:

$$
<\Delta H_{\alpha} \text { index) }>=+0.051
$$

$\sigma\left(\Delta H_{\alpha}\right.$ index $)=0.014$.

One possible cause of such a systematic difference may be a mismatch between the zero points of the $r, h, H_{\alpha}$ magnitudes used in isochrone computations and the corresponding zero points of actually observed magnitudes. Another explanation is that the adopted reddening law is not correct in the wavelength interval between the $r$ and $i$ bands and hence the computed $\mathrm{H}_{\alpha}$ index is not extinction independent. However, in this latter case there should be a trend of $\Delta \mathrm{H}_{\alpha}$-index with extinction $E_{B-V}$, which is actually absent (see Fig. 13). Hence the first explanation seems more plausible and therefore the same $\Delta \mathrm{H}{ }_{\alpha}$-index value $\left(<\Delta H_{\alpha}\right.$ index $\left.>=+0.051\right)$ can be used in calibration (3)(4) in Section 3.2 above.

\section{CONCLUSIONS}

We propose a version of the Q-method adapted for determining the extinction and distances for young open clusters based on $\mathrm{H}_{\alpha}$ ri photometry from the IPHAS survey. The method uses the coeval nature of the cluster and the fact that cluster stars are concentrated in a small space and sky area and is highly robust against age and metallicity variations over a wide range spanning $\log (t)=6.5-8.5$ and $[\mathrm{Fe} / \mathrm{H}]=-0.5$ to +0.5 . The colour excesses and distances for 18 well-studied clusters determined using our method agree well with the most bona fide published values inferred using the classical technique based on the analysis of $U B V$ photometric data. However, our age estimates agree rather poorly with the results published by other authors because of the lack of bright evolved stars in our diagrams due to the saturation of bright-star images in IPHAS frames. We found that the observed extinction-free Q-indices $\mathrm{H}_{\alpha}$ index $=0.755 r+0.245 i-H_{\alpha}$ are systematically greater than the theoretical values by $\Delta \mathrm{H}_{\alpha}$-index $=0.051 \pm 0.014$ and that this offset is practically the same for all the 18 clusters studied and shows no trend with reddening. We interpret this discrepancy as due to some mismatch between the actual and theoretical zero points of the photometric system. After correction for the inferred $\Delta \mathrm{H}_{\alpha}$-index offset the $\mathrm{H}_{\alpha}$-indices of stars with $(r-i)_{0}>0.1$ and ages in the $\log (t)=7.0-8.5$ interval can be used to determine rather accurate individual extinction values and hence to study clusters with variable extinction across their field. The results obtained show that our technique is quite well suited for determination of open cluster parameters within broad solar neighbourhood.

\section{ACKNOWLEDGEMENTS}

We thank the anonymous reviewer for the valuable comments, which greatly improved the final version of the paper. This paper makes use of data obtained as part of the INT Photometric $\mathrm{H}_{\alpha}$ Survey of the Northern Galactic Plane (IPHAS, www.iphas.org) carried out at the Isaac Newton Telescope (INT). The INT is operated on the island of La Palma by the Isaac Newton Group in the Spanish Observatorio del Roque de los Muchachos of the Instituto de Astrofisica de Canarias. All IPHAS data are processed by the Cambridge Astronomical Survey Unit, at the Institute of Astronomy in Cambridge. The bandmerged DR2 catalogue was assembled at the Centre for Astrophysics Research, University of Hertfordshire, supported by STFC grant ST/J001333/1. This research was made possible through the use of the AAVSO Photometric All-Sky Survey (APASS), funded by the Robert Martin Ayers Sciences Fund. The algorithm and new technique of the determination of fundamental open-cluster parameters was supported by the Russian Scientific Foundation (grant no. 1422-00041), and data mining and determination of the parameters of new clusters, by the joint grant by the Russian Foundation for Basic Research and Department of Science and Technology of India through project no. RFBR 15-5245121 and INT/RUS/RFBR/P-219. 
Table 3. Comparison of extinction and distance estimates determined in this paper for "standard" clusters with earlier published values

\begin{tabular}{|c|c|c|c|c|c|c|c|c|}
\hline \multirow{2}{*}{$\begin{array}{l}\text { Reference } \\
\text { PN06 }\end{array}$} & \multirow{2}{*}{$\begin{array}{r}E_{B-V^{-}} \\
E_{B-V}(\mathrm{Publ}) \\
-0.027 \\
\pm 0.022\end{array}$} & \multicolumn{2}{|c|}{$\begin{array}{c}<\mathrm{d} / \mathrm{d}(\mathrm{Publ})>\quad \sigma(\mathrm{d} / \mathrm{d}(\mathrm{Publ})) \\
(r-i, \mathrm{r}\end{array}$} & \multicolumn{2}{|c|}{$\begin{array}{c}<\mathrm{d} / \mathrm{d}(\mathrm{Publ})>\sigma(\mathrm{d} / \mathrm{d}(\mathrm{Publ})) \\
\left(\mathrm{H}_{\alpha} \text { index }, r\right)\end{array}$} & \multicolumn{2}{|c|}{$\begin{array}{c}<\mathrm{d} / \mathrm{d}(\mathrm{Publ})>\sigma(\mathrm{d} / \mathrm{d}(\mathrm{Publ})) \\
\left(\mathrm{H}_{\alpha} \text { index }, W\right)\end{array}$} & \multirow{2}{*}{$\begin{array}{r}\begin{array}{r}\text { Number } \\
\text { of clusters }\end{array} \\
5\end{array}$} \\
\hline & & $\begin{array}{r}1.12 \\
\pm \quad 0.08\end{array}$ & 0.19 & $\begin{array}{r}1.11 \\
\pm \quad 0.10\end{array}$ & 0.22 & $\begin{array}{r}1.09 \\
\pm \quad 0.07\end{array}$ & 0.16 & \\
\hline $\begin{array}{l}\text { PN06 } \\
\text { without } \\
\text { NGC } 6834\end{array}$ & $\begin{array}{r}-0.011 \\
\pm 0.021\end{array}$ & $\begin{array}{r}1.04 \\
\pm \quad 0.02\end{array}$ & 0.04 & $\begin{array}{r}1.03 \\
\pm \quad 0.07\end{array}$ & 0.13 & $\begin{array}{r}1.02 \\
\pm \quad 0.02\end{array}$ & 0.04 & 4 \\
\hline PJ94 & $\begin{array}{r}+0.011 \\
\pm 0.019\end{array}$ & $\begin{array}{r}0.89 \\
\pm \quad 0.02\end{array}$ & 0.07 & $\begin{array}{r}0.89 \\
\pm \quad 0.02\end{array}$ & 0.07 & $\begin{array}{r}0.90 \\
\pm \quad 0.02\end{array}$ & 0.07 & 13 \\
\hline M93 & $\begin{array}{r}+0.024 \\
\pm 0.042\end{array}$ & $\begin{array}{r}0.95 \\
+\quad 0.01\end{array}$ & 0.03 & $\begin{array}{r}0.89 \\
\pm \quad 0.01\end{array}$ & 0.02 & $\begin{array}{r}0.96 \\
\pm \quad 0.01\end{array}$ & 0.01 & 3 \\
\hline L14 & $\begin{array}{l}+0.025 \\
\pm 0.013\end{array}$ & $\begin{array}{r}0.92 \\
\pm \quad 0.02\end{array}$ & 0.03 & $\begin{array}{r}0.88 \\
\pm \quad 0.07\end{array}$ & 0.10 & $\begin{array}{r}0.92 \\
\pm \quad 0.02\end{array}$ & 0.03 & 2 \\
\hline K05 & -0.024 & 1.07 & - & 1.03 & - & 1.07 & - & 1 \\
\hline 3D map & $\begin{array}{r}+0.081 \\
\pm 0.006\end{array}$ & - & - & & & & & 18 \\
\hline
\end{tabular}

PN06: Paunzen and Netopil (2006); L14: Lata et al. (2014); DAML02: Dias et al. (2002); M93: Meynet et al. (1993); PJ94: Phelps and Janes (1994); K05: Keller et al. (2005); 3D map: Sale et al. (2014)

Table 4. Comparison of age estimates determined in this paper for "standard" clusters with earlier published values .

\begin{tabular}{|c|c|c|c|c|c|}
\hline Reference & $\begin{array}{r}<\log (t)-\log (\mathrm{t}(\mathrm{Publ}))> \\
\left(r-i, \mathrm{H}_{\alpha} \text { index }\right)\end{array}$ & $\sigma$ & $\begin{array}{r}<\log (t)-\log (\mathrm{t}(\mathrm{Publ}))> \\
\left(\mathrm{H}_{\alpha} \text { index } W\right)\end{array}$ & $\sigma$ & $\begin{array}{r}\text { Number } \\
\text { of clusters }\end{array}$ \\
\hline PN06 & $\begin{array}{r}+0.30 \\
\pm \quad 0.18\end{array}$ & 0.40 & $\begin{array}{r}+0.36 \\
\pm 0.20\end{array}$ & 0.45 & 5 \\
\hline PJ94 & $\begin{array}{r}+0.39 \\
\pm \quad 0.08\end{array}$ & 0.28 & $\begin{array}{r}+0.52 \\
\pm \quad 0.10\end{array}$ & 0.35 & 13 \\
\hline M93 & $\begin{array}{r}+0.23 \\
\pm \quad 0.07\end{array}$ & 0.11 & $\begin{array}{r}+0.61 \\
\pm \quad 0.22\end{array}$ & 0.39 & 3 \\
\hline L14 & $\begin{array}{r}+0.32 \\
\pm \quad 0.01\end{array}$ & 0.02 & $\begin{array}{r}+0.21 \\
\pm \quad 0.09\end{array}$ & 0.13 & 2 \\
\hline K05 & +0.14 & - & +0.18 & - & 1 \\
\hline
\end{tabular}

PN06: Paunzen and Netopil (2006); L14: Lata et al. (2014); DAML02: Dias et al. (2002); M93: Meynet et al. (1993); PJ94: Phelps and Janes (1994); K05: Keller et al. (2005); 3D map: Sale et al. (2014)

\section{REFERENCES}

Andrae R., 2010, arXiv:1009.2755

Barentsen G., Farnhill H.J., Drew J.E., et al., 2014, MNRAS, 444, 3230

Bragaglia A., Tosi M., 2006, AJ, 131, 1544

Bressan A., Marigo P., Girardi L., et al. 2012, MNRAS, 427, 127

Caetano T.C., Dias W.S., Lepine J.R.D., et al., 2015, New
Astronomy, 38, 31

Caffau E., Ludwig H.-G., Steffen M., Freytag B., Bonifacio P., 2011, Sol. Phys., 268, 255

Cardelli J.A., Clayton G.C., Mathis J.S., 1989, ApJ, 345, 245

Chen Y., Girardi L., Bressan A., Marigo P., Barbieri M., Kong Xu, 2014, MNRAS, 444, 2525

Chen Y., Bressan A., Girardi L., Marigo P., Kong Xu, Lanza A., 2015, MNRAS, 452, 1068 
Table 5. $\mathrm{H}_{\alpha}$ index offset ( $\Delta \mathrm{H}_{\alpha}$-index ) estimates for "standard" clusters.

\begin{tabular}{lrrrr} 
Cluster & $\begin{array}{r}\Delta \mathrm{H}_{\alpha} \text { index } \\
\left(r-i, \mathrm{H}_{\alpha} \text { index }\right)\end{array}$ & $\begin{array}{r}\Delta \mathrm{H}_{\alpha} \text { index } \\
\left(\mathrm{H}_{\alpha} \text { index, } r\right)\end{array}$ & $\begin{array}{r}\Delta \mathrm{H}_{\alpha} \text { index } \\
\left(\mathrm{H}_{\alpha} \text { index, } W\right)\end{array}$ & $\begin{array}{r}\text { Average per cluster } \\
<\Delta \mathrm{H}_{\alpha} \text { index }>\end{array}$ \\
\hline Be 7 & $+0.051 \pm 0.003$ & $+0.044 \pm 0.002$ & $+0.046 \pm 0.004$ & $\mathbf{+ 0 . 0 4 6} \pm \mathbf{0 . 0 0 2}$ \\
Be 62 & $+0.060 \pm 0.006$ & $+0.059 \pm 0.003$ & $+0.066 \pm 0.005$ & $\mathbf{+ 0 . 0 6 1} \pm \mathbf{0 . 0 0 2}$ \\
King 12 & $+0.081 \pm 0.004$ & $+0.085 \pm 0.002$ & $+0.075 \pm 0.003$ & $\mathbf{+ 0 . 0 8 2} \pm \mathbf{0 . 0 0 2}$ \\
NGC 103 & $+0.059 \pm 0.006$ & $+0.042 \pm 0.003$ & $+0.051 \pm 0.003$ & $\mathbf{+ 0 . 0 4 4} \pm \mathbf{0 . 0 0 2}$ \\
NGC 129 & $+0.066 \pm 0.008$ & $+0.055 \pm 0.004$ & $+0.050 \pm 0.003$ & $\mathbf{+ 0 . 0 5 3} \pm \mathbf{0 . 0 0 2}$ \\
NGC 436 & $+0.060 \pm 0.002$ & $+0.058 \pm 0.002$ & $+0.053 \pm 0.003$ & $\mathbf{+ 0 . 0 5 8} \pm \mathbf{0 . 0 0 1}$ \\
NGC 457 & $+0.039 \pm 0.003$ & $+0.026 \pm 0.002$ & $+0.032 \pm 0.002$ & $\mathbf{+ 0 . 0 3 1} \pm \mathbf{0 . 0 0 1}$ \\
NGC 581 & $+0.034 \pm 0.003$ & $+0.022 \pm 0.007$ & $+0.027 \pm 0.005$ & $\mathbf{+ 0 . 0 3 1} \pm \mathbf{0 . 0 0 2}$ \\
NGC 654 & $+0.042 \pm 0.006$ & $+0.035 \pm 0.003$ & $+0.035 \pm 0.003$ & $\mathbf{+ 0 . 0 3 6} \pm \mathbf{0 . 0 0 2}$ \\
NGC 659 & $+0.026 \pm 0.007$ & $+0.034 \pm 0.003$ & $+0.031 \pm 0.004$ & $\mathbf{+ 0 . 0 3 2} \pm \mathbf{0 . 0 0 2}$ \\
NGC 663 & $+0.059 \pm 0.007$ & $+0.059 \pm 0.003$ & $+0.058 \pm 0.002$ & $\mathbf{+ 0 . 0 5 8} \pm \mathbf{0 . 0 0 2}$ \\
NGC 869 & $+0.047 \pm 0.005$ & $+0.045 \pm 0.003$ & $+0.042 \pm 0.006$ & $\mathbf{+ 0 . 0 4 5} \pm \mathbf{0 . 0 0 2}$ \\
NGC 884 & $+0.058 \pm 0.004$ & $+0.042 \pm 0.002$ & $+0.043 \pm 0.003$ & $\mathbf{+ 0 . 0 4 5} \pm \mathbf{0 . 0 0 2}$ \\
NGC 6834 & $+0.084 \pm 0.006$ & $+0.076 \pm 0.003$ & $+0.077 \pm 0.002$ & $\mathbf{+ 0 . 0 7 7} \pm \mathbf{0 . 0 0 2}$ \\
NGC 6871 & $+0.058 \pm 0.003$ & $+0.059 \pm 0.004$ & $+0.059 \pm 0.003$ & $\mathbf{+ 0 . 0 5 9} \pm \mathbf{0 . 0 0 2}$ \\
NGC 7790 & $+0.049 \pm 0.004$ & $+0.043 \pm 0.004$ & $+0.044 \pm 0.004$ & $\mathbf{+ 0 . 0 4 5} \pm \mathbf{0 . 0 0 2}$ \\
Stock 24 & $+0.045 \pm 0.004$ & $+0.056 \pm 0.003$ & $+0.050 \pm 0.004$ & $\mathbf{+ 0 . 0 5 2} \pm \mathbf{0 . 0 0 2}$ \\
Trumpler 1 & $+0.034 \pm 0.025$ & $+0.049 \pm 0.006$ & $+0.041 \pm 0.006$ & $\mathbf{+ 0 . 0 4 5} \pm \mathbf{0 . 0 0 4}$ \\
\hline Mean & $\mathbf{+ 0 . 0 5 3} \pm \mathbf{0 . 0 0 1}$ & $\mathbf{+ 0 . 0 5 1} \pm \mathbf{0 . 0 0 1}$ & $\mathbf{+ 0 . 0 5 1} \pm \mathbf{0 . 0 0 1}$ & $\mathbf{+ 0 . 0 5 1} \pm \mathbf{0 . 0 0 1}$ \\
\hline
\end{tabular}

Dias W.S., Alessi B.S., Moitinho J.R., and Lepine J.R.D., 2002, A\&A, 389, 871

Dias W.S., Monteiro H., Caetano T.C., and Oliveira A.F., 2012, A\&A, 539, 125

Drew J.E., Greimel R., Irwin I.M., et al., 2005, MNRAS, 362,753

Efron B., 1979, Ann. Statist., 7, 1

Girardi L., Bertelli G., Bressan A., et al., 2002, A\&A, 391, 195

Gonzalez-Solares E. A. et al. 2008, MNRAS, 388,89

Hastie T., Tibshirani R., \& Friedman J. 2009, The Elements of Statistical Learning: Data Mining, Inference, and Prediction. (Springer-Verlag)

Henden A. \& Munari U. 2014, Contributions of the Astronomical Observatory Skalnate Pleso, 43, no. 3, p. 518.

Henden A. A., Templeton M., Terrell, D., et al. 2016, VizieR Online Data Catalog, II/336

Jeffries R. D., Oliveira J. M., Naylor T., Mayne N. J., Littlefair S. P., 2007, MNRAS, 376, 580

Johnson H. L., Morgan W. W., 1953, ApJ, 117, 313

Joshi Y.C., 2007, MNRAS, 378, 768

Joshi Y. C., Dambis A. K., Pandey A. K., Joshi S., 2016, A\&A, 593, 116

Keller S.C., Grebel E.K., Miller G.J, and Yoss K.M., 2001, AJ, 122, 248

Kharchenko N.V., Piskunov A.E., Schilbach E., et al., 2013, A\&A, 558, 53

Lata S., Pandey A. K., Sharma S., et al., 2014, New Astronomy, 26, 77

Mayne N. J., Naylor T., Littlefair S. P., Saunders Eric S., Jeffries R. D., 2007, MNRAS, 375, 1220

McBride V.A, Coe M. J., Negueruela I., Schurch M. P. E., McGowan K. E., 2008, MNRAS 388, 1198

Meynet G., Mermilliod J.-C., and Maeder A., 1993, A\&AS, 98, 477

Naylor T., 2009, MNRAS, 399, 432

Naylor T. and Jeffries 2006, MNRAS, 373, 1251

Netopil M., Paunzen E., and Carraro G., 2015, A\&A, 582,
19

Paunzen E. and Netopil M., 2006, MNRAS, 371, 1641

Phelps R.L. and Janes K.A., 1994, ApJS, 90, 31

Perren G. I., Vazquez R. A., Piatti A. E., 2015, A\&A, 576, 6

Popescu B., Hanson M. M., Borissova J., Kurtev R., Ivanov V. D., Catelan M., Larsen S. S., Minniti, D., Lucas P., 2014, XIV Latin American Regional IAU Meeting (Eds. A. Mateus, J. Gregorio-Hetem \& R. Cid Fernandes) Revista Mexicana de Astronomia y Astrofisica (Serie de Conferencias), 44, 163

Sale S.E., Drew J.E., Barentsen G., et al., 2014, MNRAS, 443, 2907

Schmidt-Kaler T. 1982, in Landolt-Bornstein, Group VI, Vol. 2b, Stars and Star Clusters (Berlin: Springer-Verlag)

Sung H., Lim B., Bessell M.S., et al., 2013, Journal of Korean Astronomical Society, 46, 103

Tang J., Bressan A., Rosenfield P., Slemer A., Marigo P., Girardi Leo, Bianchi L., 2014, MNRAS, 445, 4287

Yadav R.K.S., Sagar, R., 2001, MNRAS, 328, 370 